\title{
A Traffic-Flow Model with Constraints for the Modeling of Traffic Jams
}

\author{
F. Berthelin* \\ P. Degond ${ }^{\dagger}$ \\ V. Le Blanc ${ }^{\ddagger}$ \\ S. Moutari* \\ M. Rascle* \\ J. Royer ${ }^{\S}$
}

\begin{abstract}
Recently, Berthelin et al [5] introduced a traffic flow model describing the formation and the dynamics of traffic jams. This model which consists of a Constrained Pressureless Gas Dynamics system assumes that the maximal density constraint is independent of the velocity. However, in practice, the distribution of vehicles on a highway depends on their velocity. In this paper we propose a more realistic model namely the Second Order Model with Constraint (SOMC model), derived from the Aw \& Rascle model [1] abd which takes into this feature. Moreover, when the maximal density constraint is saturated, the SOMC model "relaxes" to the Lighthill \& Whitham model [17]. We prove an existence result of weak solutions for this model by means of cluster dynamics in order to construct a sequence of approximations and we solve completely the associated Riemann problem.
\end{abstract}

Key words. Traffic flow models, Second order models, Constraints, Riemann problem, Weak solutions.

AMS Subject Classification: 35L60, 35L65, 35L67

\section{Introduction}

During the past fifty years, a wide range of models of vehicular traffic flow has been developed. Roughly speaking, three important classes of approaches are commonly used to model traffic phenomena. (i) Microscopic models or Carfollowing models e.g. [11, 2]: they are based on supposed mechanisms describing the process of one vehicle following another; (ii) Kinetic models [22, 20, 19, 14, 18, 13]: they describe the dynamics of the velocity distribution of vehicles, in the traffic flow; (iii) Fluid-dynamical models [17, 23, 21, 9, 22, 1, 25, 6, 8, 5, 4]:

${ }^{*}$ Laboratoire J. A. Dieudonné, UMR CNRS No 6621, Université de Nice-Sophia Antipolis, Parc Valrose, 06108 Nice Cedex 2, France. \{bertheli, salissou, rascle\}@math.unice.fr

${ }^{\dagger}$ Laboratoire MIP, Université Paul Sabatier Toulouse III, 31062 Toulouse Cedex 9, France. degond@mip.ups-tlse.fr

${ }^{\ddagger}$ Laboratoire UMPA, UMR CNRS No 5669, ENS de Lyon, 46 Allée d’Italie, 69364 Lyon Cedex 07, France. valerie.le.blanc@umpa.ens-lyon.fr

$\S$ Laboratoire de Mathématiques Jean Leray UMR CNRS No 6629, UFR Sciences et Techniques 2 Rue de la Houssinière - BP 92208 F-44322 Nantes Cedex 3, France. julien.royer@enslyon.org 
they describe the dynamics of macroscopic variables (e.g. density, velocity, and flow) in space and time.

Here, we are concerned with the latter approch, i.e., the fluid-dynamical models. The first fluid model is due to Lighthill and Whitham [17] and Richards [23]. It consists of a single equation, the continuity one, thereby it is called "first order" model. Since then, various modifications and extensions to this basic model have been proposed in the literature. At the same time, nonequilibrium "second order" models, which consist of the continuity equation coupled with another equation describing the acceleration behaviour, have been developed. They are based either on perturbations of the isentropic gas dynamics models, see e.g. $[21,15,12]$, or on heuristic considerations and a derivation from the Follow-the-Leader model (FLM) [1, 5, 6, 8, 25].

In this paper, we propose a second order model, called the Second Order Model with Constraints (SOMC), which we derived from the Aw \& Rascle (AR) model [1] through a singular limit. We prove an existence result of weak solutions for such a model and discuss the associated Riemann problem. In contrast with the model introduced in [5] which is rather crude, as it assumes that the maximal density is constant (therefore independent of the velocity), here, we take into account the dependence of the maximal density constraint on the velocity. This consideration leads to a more realistic formulation, since it is well known that in practice, the distribution of vehicles on a highway, depends on their velocity. Furthermore, the particularity of the model we propose here, is its double-sided behaviour. Indeed, when the density constraint is saturated i.e., the maximal density is attained, for a given velocity, the SOMC model behaves like the Lighthill \& Whitham first order model, whereas in the free flow our model behaves like the pressureless gas model. Moreover, even in the Riemann problem, the interaction between two constant states in either regime can produce new states in the other regime: in other words the two regimes are intimately coupled and thus cannot ignore each other. Due to this specific property, we expect our model to capture some traffic complex phenomena such as stop and go waves.

The remaining parts of the paper are organized as follows. In Section 2, we first introduce the SOMC model, justify its motivations, then we outline and discuss sufficient conditions for its derivation from the Aw \& Rascle second order model [1]. Section 3 provides an existence result of weak solutions to the SOMC system. The Riemann Problem for the SOMC model is completely discussed in Section 4 . We finally conclude with directions for further research in Section 5.

\section{The model and its derivation}

In this section, we present the Second Order Model with Constraint and highlight its specific properties. We justify the motivations of this model and discuss its derivation from the Aw \& Rascle second order model [1]. 


\subsection{The Second Order Model with Constraint (SOMC)}

The second order model we introduce in this paper, namely the Second Order Model with Constaint (SOMC), writes

$$
\begin{aligned}
& \partial_{t} n(x, t)+\partial_{x}(n(x, t) u(x, t))=0, \\
& \left(\partial_{t}+u(x, t) \partial_{x}\right)(u+\bar{p})(x, t)=0, \\
& 0 \leq n(x, t) \leq n^{*}(u(x, t)), \quad \bar{p} \geq 0, \quad\left(n^{*}(u)-n\right) \bar{p}=0,
\end{aligned}
$$

where $n, u$ and $n^{*}(u)$ denote respectively the density, the velocity and the maximal density. The functional $\bar{p}(n, u)$ is the offset velocity between the actual velocity $u$ and the preferred velocity given by $u+\bar{p}$.

Definition 2.1. We call a cluster or a block, a stretch of road defined by an interval $\left[x_{1}(t), x_{2}(t)\right]$, inside which the system (2.34)-(2.36) is satisfied and

$n(x, t)=\left\{\begin{array}{l}n^{*}(u(x, t)), \quad \text { if } x \in\left[x_{1}(t), x_{2}(t)\right] ; \\ 0, \quad \text { if } x \in\left[x_{1}(t)-\varepsilon(t), x_{1}(t)[\cup] x_{2}(t), x_{2}(t)+\varepsilon(t)\right], \text { for } \varepsilon(t) \text { small. }\end{array}\right.$

It is well known that in traffic, the minimal distance between a driver and its leading car is an increasing function of the velocity. Therefore, in contrast with the model introduced in [5], here the maximal density $n^{*}$ is a functional of the velocity $u$. However, this natural consideration imparts to the SOMC model a particular property: a double behaviour. Indeed, when $n(x, t)=n^{*}(u(x, t))$, i.e. the maximal density constraint $n(x, t) \leq n^{*}(u(x, t))$ is saturated, a block of vehicles (or a cluster) forms. In a cluster, $u$ and $p$ are layed down by the first vehicle, and as long as the cluster is going freely, these variables remain constant, see Section 3 and the discussions in Section 4 below. Therefore, inside each cluster which is going freely, the SOMC model writes

$$
\partial_{t} n^{*}(u)+\partial_{x}\left(n^{*}(u) u\right)=0 .
$$

Let $n \longrightarrow u^{*}(n)$ be the inverse functional of $u \longrightarrow n^{*}(u)$. Therefore $(2.4)$ rewrites

$$
\partial_{t} n+\partial_{x}\left(n u^{*}(n)\right)=0
$$

where $q(n):=n u^{*}(n)$ is the flux function as in the Lighthill \& Whitham model [17]. Therefore, we have a hyperbolic second order model which "relaxes" to the Lighthill \& Whitham first order model when the maximal density constraint is saturated. Hence, the SOMC model is expected to capture the stop and go waves phenomena since there is no invariant region for the velocity $u$ when the model behaves as the Lighthill \& Whitham model.

\subsection{Derivation of the SOMC model}

This paragraph is dedicated to the derivation of the SOMC model from the Aw \& Rascle second order model [1]. For sake of completeness, we present first the classical case in which the maximal density $n^{*}$ is constant (i.e., independent of the velocity). Then, we introduce the case $n^{*}:=n^{*}(u)$ and justify its motivations. Afterwards we discuss the derivation of the SOMC model from the Aw \& Rascle model through a singular limit. 


\subsubsection{The case $n^{*}=$ constant}

In conservative form, the Aw \& Rascle (AR) macroscopic model [1] consists of the following equations

$$
\begin{aligned}
& \partial_{t} n+\partial_{x}(n u)=0, \\
& \partial_{t}(n w)+\partial_{x}(n w u)=0, \\
& w=u+p(n),
\end{aligned}
$$

where $n(x, t)(\geq 0)$ and $u(x, t)(\geq 0)$ denote respectively the local density (number of vehicles per unit of space) and the velocity, both at the position $x$ and the time $t$. The variable $w$ denotes the drivers "preferred velocity" and $0 \leq p(n) \leq \infty$ is the velocity offset between the actual velocity and the preferred velocity.

In what follows we give some important properties of the AR model and refer the reader to [1] for more details.

Let us rewrite the system (2.6)-(2.8) in the following general form

$$
\begin{gathered}
\partial_{t} U+A(U) \partial_{x} U=0 \\
\text { with } U=\left(\begin{array}{l}
n \\
u
\end{array}\right) \text { and } A(U)=\left(\begin{array}{cc}
u & n \\
0 & u-n p^{\prime}(n)
\end{array}\right) .
\end{gathered}
$$

The system (2.9)-(2.10) (or (2.6)-(2.8)) is strictly hyperbolic away from the vacuum (i.e. when $n \neq 0)$. Indeed, the eigenvalues of the jacobian matrix $A(U)$ are

$$
\lambda_{1}=u-n p^{\prime}(n) \leq \lambda_{2}=u .
$$

and the associated eigeinvectors are respectively

$$
r_{1}=\left(\begin{array}{l}
1 \\
-p^{\prime}(n)
\end{array}\right) \quad \text { and } \quad r_{2}=\left(\begin{array}{l}
1 \\
0
\end{array}\right) .
$$

The eigenvalues of the system correspond to the information propagation speed and they are both bounded by the traffic flow speed. Thereby the model complies with the anisotropic features of traffic flow.

Since $\nabla \lambda_{1} \cdot r_{1} \neq 0$ and $\nabla \lambda_{2} \cdot r_{2}=0$ (here $\left.\nabla:=\left(\partial / \partial_{n}, \partial / \partial_{n w}\right)\right)$, then $\lambda_{1}$ is genuinely nonlinear and $\lambda_{2}$ is linearly degenerate. Therefore, the waves associated to $\lambda_{1}$ correspond to shock waves (braking) or rarefaction waves (acceleration) which modify the velocity, whereas the waves associated to $\lambda_{2}$ correspond to contact discontinuities. In this model, the shock and rarefaction curves coincide, therefore the model falls into the class of "Temple Systems" [24]. The Riemann invariants in the sense of Lax [16] for the system (2.6)-(2.8) are respectively $w$ and $u$.

Naturally in the traffic dynamics, at each time $t>0$, the following constraints have to be satisfied

$$
\begin{aligned}
& 0 \leq u(., t) \leq u^{*}, \\
& 0 \leq n(., t) \leq n^{*},
\end{aligned}
$$

with $u^{*}<\infty$ and $n^{*}<\infty$ respectively the maximal velocity and density.

In the $(u, w)$ plane, this region is defined by

$$
R_{u^{*}, n^{*}}=\left\{0 \leq u \leq u^{*}, \quad 0 \leq w-u \leq p\left(n^{*}\right)\right\}
$$


which is not an invariant region for the AR model. Therefore, for some badly chosen initial data in $R_{u^{*}, n^{*}}$, one may obtain for some $(x, t)$ solutions which are later on out of the region $R_{u^{*}, n^{*}}$. There are two possible strategies to avoid the possible (unpleasant!) appearance of densities $n>n^{*}$ in the future. One consists in using invariant rectangles in the plane $(u, w)$, see [1]. The other one is to choose a velocity offset $p$ which is singular at $n=n^{*}$. One of the good candidate proposed in [5] is

$$
p(n)=\left(\frac{1}{n}-\frac{1}{n^{*}}\right)^{-\gamma} \text { with } n \leq n^{*} \text { and } \gamma>0,
$$

where $n^{*}$ denotes the maximal density. The AR model with the constraints (2.12)-(2.13) and the function $p$ given by (2.15) is called the Modified AR model (MAR). Obviously, the MAR model inherits the properties of the AR model stated above (see [5]).

It is known that drivers do not reduce significantly their speed unless they are too closed to the maximal density, what means the velocity offset $p \longrightarrow 0$ in free flow traffic. In the MAR model, this can be taken into acount by replacing the functional $p$ by the rescaled one: $\varepsilon p$ with $\varepsilon \longrightarrow 0$. Therefore the rescaled model can be stated as follows

$$
\begin{aligned}
& \partial_{t} n^{\varepsilon}+\partial_{x}\left(n^{\varepsilon} u^{\varepsilon}\right)=0, \\
& \left(\partial_{t}+u^{\varepsilon} \partial_{x}\right)\left(u^{\varepsilon}+\varepsilon p\left(n^{\varepsilon}\right)\right)=0,
\end{aligned}
$$

where $p(n)$ is defined in (2.15). The system (2.16)-(2.17) is called the Rescaled Modified Aw \& Rascle model (RMAR). Furthermore, it conserves the properties of the MAR model.

Now we recall briefly the Constrained Pressureless Gas Dynamics model and we refer the reader to [5] for more details on this model.

Due to the form of the modified velocity offset (2.15),

$$
p(n) \longrightarrow \infty \text { when } n \longrightarrow n^{*} .
$$

Assume that $\varepsilon p\left(n^{\varepsilon}\right)(x, t)$ has a limit: $\bar{p}(x, t):=\lim _{\varepsilon \longrightarrow 0} \varepsilon p\left(n^{\varepsilon}\right)(x, t)$. If $n=n^{*}$ at the point $(x, t), \bar{p}$ may become non zero and finite, and $\bar{p}$ turns out to be a Lagrangian multiplier of the constraint $n \leq n^{*}$. In others words,

$$
\bar{p}=\left\{\begin{array}{l}
0 \text { if } n<n^{*}, \\
c(0<c<\infty) \text { if } n=n^{*} .
\end{array}\right.
$$

Finally, the formal limit of the RMAR system (2.16)-(2.17) leads to the Constrained Pressureless Gas Dynamics model (CPGD):

$$
\begin{aligned}
& \partial_{t} n+\partial_{x}(n u)=0, \\
& \left(\partial_{t}+u \partial_{x}\right)(u+\bar{p})=0, \\
& 0 \leq n \leq n^{*}, \quad \bar{p} \geq 0, \quad\left(n^{*}-n\right) \bar{p}=0 .
\end{aligned}
$$

\subsubsection{The case $n^{*}=n^{*}(u)$}

As in practice, the minimal distance between a driver and its leading car is an increasing function of the velocity, a more realistic formulation of traffic flow 
must include this fact. With this consideration, the velocity offset $p$ takes the form

$$
p(n, u)=\left(\frac{1}{n}-\frac{1}{n^{*}(u)}\right)^{-\gamma}, \text { with } n \leq n^{*}(u), \text { and } \gamma>0 .
$$

With the functional $p$ in the above form (2.22) the MAR model presented above turns to

$$
\begin{aligned}
& \partial_{t} n+\partial_{x}(n u)=0, \\
& \left(\partial_{t}+u \partial_{x}\right)(u+p(n, u))=0 .
\end{aligned}
$$

From now on, $p$ and $n^{*}$ denote respectively $p(n, u)$ and $n^{*}(u)$.

Now,

$$
\begin{aligned}
0 & =\left(\partial_{t}+u \partial_{x}\right)(u+p(n, u)) \\
& =\partial_{t} u+u \partial_{x} u+\partial_{n} p \partial_{t} n+\partial_{u} p \partial_{t} u+u \partial_{n} p \partial_{x} n+u \partial_{u} p \partial_{x} u \\
& =\partial_{t} u+u \partial_{x} u-n \partial_{n} p \partial_{x} u+\partial_{u} p \partial_{t} u+u \partial_{u} p \partial_{x} u \\
& =\left(1+\partial_{u} p\right) \partial_{t} u+\left[u\left(1+\partial_{u} p\right)-n \partial_{n} p\right] \partial_{x} u,
\end{aligned}
$$

then the system (2.23)-(2.24), called the Modified $\mathrm{AR}^{*}$ model (MAR*), can be rewritten as

$$
\begin{gathered}
\partial_{t} U+A(U) \partial_{x} U=0 \\
\text { with } U=\left(\begin{array}{l}
n \\
u
\end{array}\right) \text { and } A(U)=\left(\begin{array}{cc}
u & n \\
0 & u-\frac{n \partial_{n} p}{1+\partial_{u} p}
\end{array}\right) \text {. }
\end{gathered}
$$

The eigenvalues of the matrix $A(U)$ are

$$
\lambda_{1}=u-\frac{n \partial_{n} p}{1+\partial_{u} p} \leq \lambda_{2}=u,
$$

and the associated eigenvectors are respectively

$$
r_{1}=\left(\begin{array}{c}
1+\partial_{u} p \\
-\partial_{n} p
\end{array}\right) \text { and } r_{2}=\left(\begin{array}{c}
1 \\
0
\end{array}\right) .
$$

Since $\nabla \lambda_{2} \cdot r_{2}=0$, the second eigenvalue is linearly degenerate (here, $\nabla:=$ $\left(\frac{\partial}{\partial n}, \frac{\partial}{\partial u}\right)$ ), the waves associated to $\lambda_{2}$ are contact discontinuities.

Now let us consider the first eigenvalue $\lambda_{1}$. We have 


$$
\nabla \lambda_{1}=\left(\begin{array}{c}
-\frac{\partial_{n} p}{1+\partial_{u} p}-\frac{n \partial_{n n} p}{1+\partial_{u} p}+\frac{n \partial_{n} p \partial_{u n} p}{\left(1+\partial_{u} p\right)^{2}} \\
1-\frac{n \partial_{u n} p}{1+\partial_{u} p}+\frac{n \partial_{n} p \partial_{u u} p}{\left(1+\partial_{u} p\right)^{2}}
\end{array}\right)
$$

then

$$
\nabla \lambda_{1} \cdot r_{1}=-2 \partial_{n} p+\frac{2 n \partial_{n} p \partial_{u n} p}{1+\partial_{u} p}-n \partial_{n n} p-\frac{n\left(\partial_{n} p\right)^{2} \partial_{u u} p}{\left(1+\partial_{u} p\right)^{2}} .
$$

Clearly, $\exists(n, u) \in \mathbb{R}_{+}^{*} \times \mathbb{R}_{+}^{*}$ such that $\nabla \lambda_{1} . r_{1} \neq 0$, hence $\lambda_{1}$ is not linearly degenerate. Therefore we would like $\lambda_{1}$ to be genuinely nonlinear i.e. $\nabla \lambda_{1} . r_{1} \neq$ 0 for all $(n, u) \neq(0,0)$. In fact, one can easily notice that we need some assumptions on the functional $n^{*}: u \longmapsto n^{*}(u)$.

We consider the following assumptions:

(A-1) $n^{*}(u)$ is twice continuously differentiable;

(A-2) $n^{*}(u)$ is strictly decreasing.

(A-3) $n^{*}(u)$ concave $\left(\frac{d^{2}}{d u^{2}}\left(n^{*}(u)\right) \leq 0\right)$.

The second assumption is quite natural, since the faster the vehicles the larger the spacing between them.

Lemma 2.1. Under assumptions $(\boldsymbol{A}-\mathbf{1})-(\boldsymbol{A}-3)$, the eigenvalue $\lambda_{1}$ is genuinely non linear. dix

For readability reasons, the proof of this lemma is postponed in the Appen-

Since $\lambda_{1}$ is genuinely non linear, therefore, the associated waves are either shocks or rarefaction waves. The Riemann invariants in the sense of Lax [16] associated to the eigenvalues $\lambda_{1}$ and $\lambda_{2}$ are respectively

$$
w=u+p(n, u) \text { and } z=u .
$$

For the same reason as in the previous paragraph, let us multiply by $\varepsilon$ the velocity offset $p$ in the model (2.23)-(2.24). Then, we obtain

$$
\begin{gathered}
\partial_{t} U^{\varepsilon}+A\left(U^{\varepsilon}\right) \partial_{x} U^{\varepsilon}=0 \\
\text { with } U^{\varepsilon}=\left(\begin{array}{c}
n^{\varepsilon} \\
u^{\varepsilon}
\end{array}\right) \text { and } A\left(U^{\varepsilon}\right)=\left(\begin{array}{cc}
u^{\varepsilon} & n^{\varepsilon} \\
0 & u^{\varepsilon}-\frac{\varepsilon n^{\varepsilon} \partial_{n} p}{1+\varepsilon \partial_{u} p}
\end{array}\right) .
\end{gathered}
$$

Hence the eigenvalues and the Riemann invariants in the sense of Lax [16] are respectively

$$
\lambda_{1}^{\varepsilon}=u^{\varepsilon}-\frac{\varepsilon n^{\varepsilon} \partial_{n} p}{1+\varepsilon \partial_{u} p} \leq \lambda_{2}^{\varepsilon}=u^{\varepsilon}
$$


and $w^{\varepsilon}=u^{\varepsilon}+\varepsilon p\left(n^{\varepsilon}, u^{\varepsilon}\right), z^{\varepsilon}=u^{\varepsilon}$. This modification conserves the properties of the model (2.23)-(2.24). The system (2.31)-(2.32) is the so-called Rescaled Modified $\mathrm{AR}^{*}$ Model $\left(\mathrm{RMAR}^{*}\right)$

Let $\bar{p}=\lim _{\varepsilon \longrightarrow 0} \varepsilon p\left(n^{\varepsilon}, u^{\varepsilon}\right)(x, t)$ and $\left(n^{\varepsilon}, u^{\varepsilon}\right) \underset{\varepsilon \longrightarrow 0}{\longrightarrow}(n, u)$, then the formal limit of the RMAR* model (2.31)-(2.32) is given by

$$
\begin{aligned}
& \partial_{t} n+\partial_{x}(n u)=0, \\
& \left(\partial_{t}+u \partial_{x}\right)(u+\bar{p})=0, \\
& 0 \leq n(x, t) \leq n^{*}(u(x, t)), \quad \bar{p} \geq 0, \quad\left(n^{*}(u)-n\right) \bar{p}=0,
\end{aligned}
$$

which is nothing but the SOMC model.

Proposition 2.1. Assume that $\varepsilon p\left(n^{\varepsilon}, u^{\varepsilon}\right) \underset{\varepsilon \longrightarrow 0}{\longrightarrow} \bar{p}>0, u^{\varepsilon}(x, t) \longrightarrow u$ and $n^{\varepsilon}-n^{*}\left(u^{\varepsilon}\right) \underset{\varepsilon \longrightarrow 0}{\longrightarrow} 0$. Then

$$
\lambda_{1}^{\varepsilon}=u^{\varepsilon}-\frac{\varepsilon n^{\varepsilon} \partial_{n} p}{1+\varepsilon \partial_{u} p} \underset{\varepsilon \longrightarrow 0}{\longrightarrow} u+\frac{n^{*}(u)}{\left(n^{*}\right)^{\prime}(u)}=\bar{\lambda}_{1}(u)
$$

Proof. Since $\underset{\varepsilon \longrightarrow 0}{\varepsilon p\left(n^{\varepsilon}, u^{\varepsilon}\right)}=\bar{p}>0$, then

$$
\exists \delta>0 \text { such that } \forall \varepsilon>0, \varepsilon p\left(n^{\varepsilon}, u^{\varepsilon}\right) \geq \delta .
$$

Thus we have

$$
\varepsilon \partial_{u} p\left(n^{\varepsilon}, u^{\varepsilon}\right)=-\gamma \frac{\left(n^{*}\right)^{\prime}\left(u^{\varepsilon}\right)}{n^{*}\left(u^{\varepsilon}\right)^{2}}\left(\frac{1}{n^{\varepsilon}}-\frac{1}{n^{*}\left(u^{\varepsilon}\right)}\right)^{-1} \varepsilon p\left(n^{\varepsilon}, u^{\varepsilon}\right) \underset{\varepsilon \longrightarrow 0}{\longrightarrow} \infty .
$$

Therefore,

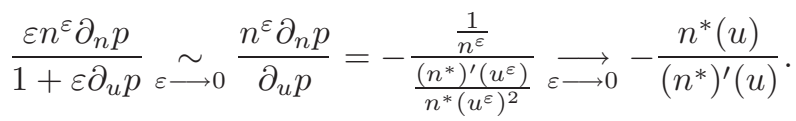

Finally

$$
\lambda_{1}^{\varepsilon} \underset{\varepsilon \longrightarrow 0}{\longrightarrow} u+\frac{n^{*}(u)}{\left(n^{*}\right)^{\prime}(u)}=\bar{\lambda}_{1}(u) .
$$

Furthermore,

$$
\frac{d \bar{\lambda}_{1}(u)}{d u}=1+\frac{\left(n^{*}\right)^{\prime}(u)^{2}-n^{*}(u)\left(n^{*}\right)^{\prime \prime}(u)}{\left(n^{*}\right)^{\prime}(u)^{2}} .
$$

Since $n^{*}(u)$ is concave, then $\bar{\lambda}_{1}(u)$ is strictly increasing. The limit $\bar{\lambda}_{1}$ is the characteristic speed of the Lighthill \& Whitham model when $n=n^{*}(u)$. Contrarily to the case $n^{*}=$ constant, here $\left|\bar{\lambda}_{1}\right|<+\infty$. In other words, a velocity variation in front of a cluster propages with a finite speed (but not with an infinite speed as in [5]) through the whole cluster. 


\section{Existence result for the SOMC model (2.34)- $(2.36)$}

This section is devoted to the proof of the existence of weak solutions to the SOMC system (2.34)-(2.36), written in conservative form. The proof is based on the results in [3] and is strongly motivated by the analysis of the Riemann problem in Section 4. Indeed, this analysis permits us to exhibit the limit as $\varepsilon \longrightarrow 0$ of the solutions to the Riemann problem of the RMAR* model (2.31)(2.32), which are nothing but the expected solutions to the Riemann problem of the SOMC model (2.34)-(2.36). For instance, when two blocks collide i.e., the cluster behind is going faster than the cluster ahead, a shock wave appears at the front of the cluster behind and propagates upstream with a finite speed. This technical and "self contained" analysis is postponed at the end of the paper for readability reasons. However, it is not needless since it justfies the choice of the dynamics considered below, and allows us to expect that the obtained solution (non unique) is the one which models the real phenomena. First we prove the existence of weak solutions for some particular data and then we prove the stability of the obtained solutions. Namely, we make use of the result in [3], in which it has been proved that any smooth function can be approximated in the distribution sense by a sequence of characteristic functions.

In conservative form, the SOMC model (2.34)-(2.36) is written as follows

$$
\begin{aligned}
& \partial_{t} n+\partial_{x}(n u)=0, \\
& \partial_{t}(n(u+\bar{p}))+\partial_{x}(n(u+\bar{p}) u)=0, \\
& 0 \leq n(x, t) \leq n^{*}(u(x, t)), \quad \bar{p} \geq 0, \quad\left(n^{*}(u)-n\right) \bar{p}=0
\end{aligned}
$$

\subsection{Clusters dynamics}

In order to prove the existence of solutions for the SOMC model, we mimic the approach of [7] (which was also used in [5]). We approximate the initial datum as a succession of vacuum and blocks (or clusters) where the constraint is saturated. Physically, this means that any traffic condition can be approximated in the weak sense by a situation where saturated stretches of road are followed by empty stretches. So, our first task is to consider the dynamics of a solution which consists of a succession of clusters and vacuum. In particular, the key point in defining this dynamics is to specify what happens when a faster cluster meets a slower one in front. To define what happens when two clusters meet, we take inspiration from the examination of the solutions of the Riemann problem, which is developed in Section 4. In what follows, we construct the cluster (or block) solutions to (3.1a)-(3.1c).

Now let us consider the density $n(x, t)$, the flux $n(x, t) u(x, t)$ and the quantity $n(x, t) \bar{p}(x, t)$ given respectively by 


$$
\begin{aligned}
& n(x, t)=\sum_{i=1}^{N} n_{i}^{*}(t) \mathbb{I}_{a_{i}(t)<x<b_{i}(t)}, \\
& n(x, t) u(x, t)=\sum_{i=1}^{N} n_{i}^{*}(t) u_{i}(t) \mathbb{I}_{a_{i}(t)<x<b_{i}(t)} \\
& n(x, t) \bar{p}(x, t)=\sum_{i=1}^{N} n_{i}^{*}(t) \bar{p}_{i}(t) \mathbb{I}_{a_{i}(t)<x<b_{i}(t)}
\end{aligned}
$$

with $n_{i}^{*}(t)=n^{*}\left(u_{i}(t)\right)$ (or equivalently $u_{i}=u^{*}\left(n_{i}\right)$ ) as long as there is no collision. That is to say $a_{N}(t)<b_{N}(t)<a_{N-1}(t)<b_{N-1}(t)<\ldots<b_{1}(t)$ and the number of blocks $N$ is constant until there is a shock.

If there is no collision, each block $i$ moves freely with a constant velocity, i.e. $u_{i}(t):=u_{i}$. Therefore $n_{i}^{*}(t)=n^{*}\left(u_{i}\right):=n_{i}^{*}$ is also constant. On the other hand, when a block $i+1$ catches up with the block ahead $i$ at time $t^{*}$ (that implies in particular that $u_{i+1}>u_{i}$ ), then a shock wave appears and propagates gradually inside the block $i+1$. The shock speed $u_{s}$ is given by

$$
u_{s}=\frac{n_{i}^{*} u_{i}-n_{i+1}^{*} u_{i+1}}{n_{i}^{*}-n_{i+1}^{*}}
$$

We notice that since $u_{i+1}>u_{i}$ then necessarily $n_{i}^{*} \neq n_{i+1}^{*}$.

The dynamics is illustrated by Figure 1.

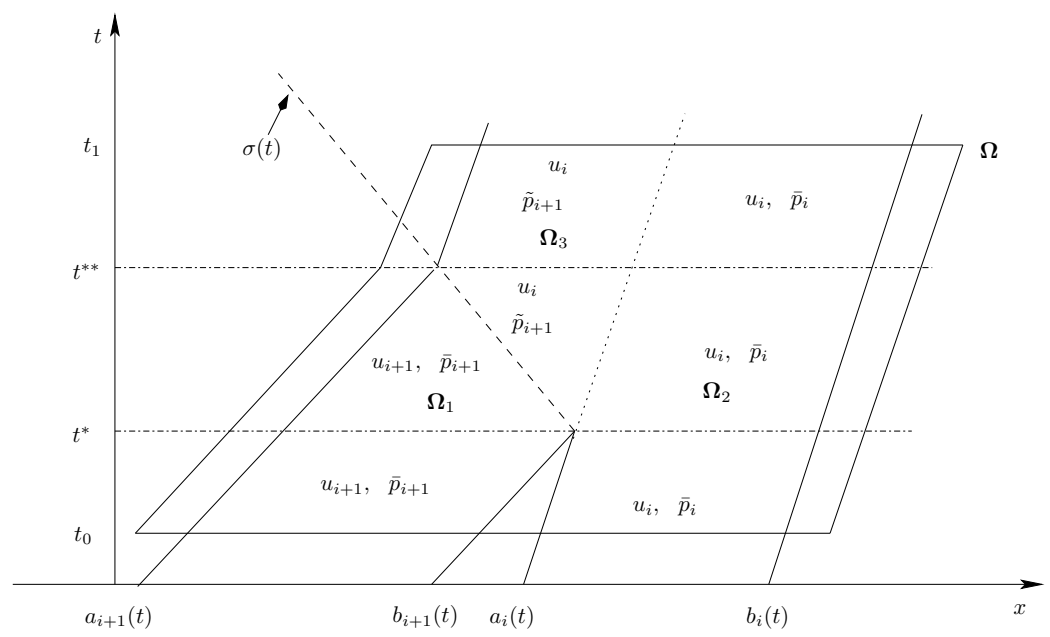

Figure 1: The traffic dynamics when two blocks collide.

Let $\sigma(t)$ be the wave trajectory i.e. $\sigma^{\prime}(t)=u_{s}$. In contrast to [5], here the block $(i+1)$ does not take instantanously the velocity $u_{i}$ but it adjusts its velocity gradually through $\sigma(t)$. Let $t^{* *}$ be the time such that $\sigma(t)$ reaches the left boundary of the block $i+1$, see Figure 1. Around this shock, the density $n(x, t)$, the flux $n(x, t) u(x, t)$ and the functional $\bar{p}(x, t)$ are locally given respectively by 


$$
\begin{aligned}
& n(x, t)=\left\{\begin{array}{lr}
n_{i}^{*} \mathbb{I}_{a_{i}(t)<x<b_{i}(t)}+n_{i+1}^{*} \mathbb{I}_{a_{i+1}(t)<x<b_{i+1}(t)} & \text { if } t<t^{*}, \\
n_{i}^{*} \mathbb{I}_{a_{i}(t)<x<b_{i}(t)}+n_{i}^{*} \mathbb{I}_{\sigma(t)<x<a_{i}(t)} & \text { if } t^{*}<t<t^{* *}, \\
+n_{i+1}^{*} \mathbb{I}_{a_{i+1}(t)<x<\sigma(t)} & \text { if } t>t^{* *},
\end{array}\right.
\end{aligned}
$$

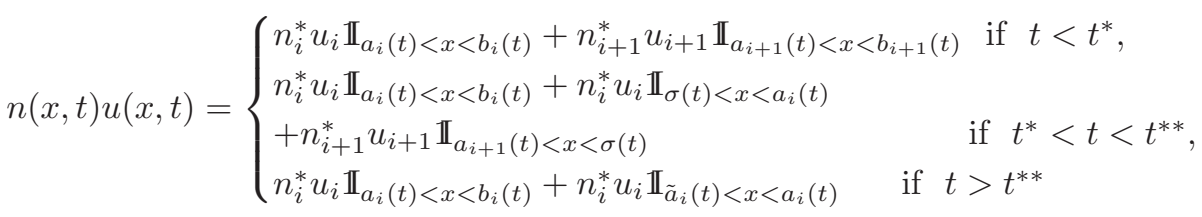

and

$$
n(x, t) \bar{p}(x, t)=\left\{\begin{array}{l}
n_{i}^{*} \bar{p}_{i} \mathbb{I}_{a_{i}(t)<x<b_{i}(t)}+n_{i+1}^{*} \bar{p}_{i+1} \mathbb{I}_{a_{i+1}(t)<x<b_{i+1}(t)} \\
n_{i}^{*} \bar{p}_{i} \mathbb{I}_{a_{i}(t)<x<b_{i}(t)}+n_{i}^{*} \tilde{p}_{i+1} \mathbb{I}_{\sigma(t)<x<a_{i}(t)} \quad \text { if } t<t^{*}, \\
+n_{i+1}^{*} \bar{p}_{i+1} \mathbb{I}_{a_{i+1}(t)<x<\sigma(t)} \quad \text { if } t^{*}<t<t^{* *}, \\
n_{i}^{*} \bar{p}_{i} \mathbb{I}_{a_{i}(t)<x<b_{i}(t)}+n_{i}^{*} \tilde{p}_{i+1} \mathbb{I}_{\tilde{a}_{i}(t)<x<a_{i}(t)} \quad \text { if } t>t^{* *},
\end{array}\right.
$$

with

$$
\tilde{p}_{i+1}=\bar{p}_{i+1}+u_{i+1}-u_{i} \geq 0,
$$

and

$$
\tilde{a}_{i}^{\prime}=u_{i} \text {, and } \tilde{a}_{i}\left(t^{* *}\right)=\sigma\left(t^{* *}\right) .
$$

Remark 3.1. The velocity $u$ and the "pressure" $\bar{p}$ are assumed to be extended linearly in the vacuum $(n=0)$ between two successive blocks. Moreover we assume that $u$ and $\bar{p}$ are constant at $\pm \infty$.

\subsection{Properties of the cluster dynamics}

Let us start this section by the following result.

Theorem 3.1. With the above dynamics, the quantities $n(x, t), u(x, t)$ and $\bar{p}(x, t)$ defined by (3.2)-(3.4) and Remark 3.1 are solutions to (3.1a)-(3.1c).

Proof. When there is no collision, each block $i$ moves freely at a constant velocity $u_{i}(t):=u_{i}$. The density $n_{i}^{*}(t)=n^{*}\left(u_{i}\right):=n_{i}^{*}$ and the "pressure" $\bar{p}_{i}(t)$ are also constant in each block $i$. Then, $(n, u, \bar{p})$ defined by (3.2)-(3.3)-(3.4) solves the system (3.1a)-(3.1c). Now let us turn to the case of collision of two blocks at time $t^{*}$ in the above dynamics. Let $\Omega$ be a domain which only contains the two blocks concerned with this collision, see Figure 1 . Then $\Omega$ is given by

$$
\Omega=\Omega_{1} \cup \Omega_{2} \cup \Omega_{3},
$$


where

$$
\begin{aligned}
\Omega_{1}= & \left\{(x, t) ; a_{i+1}(t) \leq x \leq b_{i+1}(t) \text { and } t_{0}<t \leq t^{*}\right\} \\
& \cup\left\{(x, t) ; a_{i+1}(t) \leq x \leq \sigma(t) \text { and } t^{*} \leq t \leq t^{* *}\right\} \\
\Omega_{2}= & \left\{(x, t) ; a_{i}(t) \leq x \leq b_{i}(t) \text { and } t_{0}<t \leq t^{*}\right\} \\
& \cup\left\{(x, t) ; a_{i}(t) \leq x \leq b_{i}(t) \text { and } t^{*} \leq t \leq t^{* *}\right\} \\
& \cup\left\{(x, t) ; a_{i}(t) \leq x \leq b_{i}(t) \text { and } t^{* *} \leq t \leq t_{1}\right\} \\
\Omega_{3}= & \left\{(x, t) ; \sigma(t) \leq x \leq a_{i}(t) \text { and } t^{*} \leq t \leq t^{* *}\right\} \\
& \cup\left\{(x, t) ; \tilde{a}_{i}(t) \leq x \leq a_{i}(t) \text { and } t^{* *} \leq t \leq t_{1}\right\},\left(\text { with } \tilde{a}_{i}(t) \equiv a_{i+1}(t)\right) .
\end{aligned}
$$

Let $\varphi(x, t)$ be a smooth function with compact support in $\Omega$. For any continuous function $S$, denoting by $<,>$ the distribution duality brackets, we have

$$
\begin{aligned}
<\partial_{t}(n S(u, \bar{p}))+\partial_{x}(n u S(u, \bar{p})), \varphi> & =-\iint_{\Omega} n S(u, \bar{p})\left(\partial_{t} \varphi+u \partial_{x} \varphi\right) d x d t \\
& =A_{1}+A_{2}+A_{3}
\end{aligned}
$$

where $A_{j}=\iint_{\Omega_{j}} n S(u, \bar{p})\left(\partial_{t} \varphi+u \partial_{x} \varphi\right) d x d t$, for $j=1 \ldots 3$.

For all $i=1 \ldots N$, we have

$$
\int_{a_{i}(t)}^{b_{i}(t)} \partial_{x} \varphi(x, t) d x=\varphi\left(b_{i}(t), t\right)-\varphi\left(a_{i}(t), t\right)
$$

On the other hand

$$
\int_{a_{i}(t)}^{b_{i}(t)} \partial_{t} \varphi(x, t) d x=\frac{d}{d t}\left[\int_{a_{i}(t)}^{b_{i}(t)} \varphi(x, t) d x\right]-\varphi\left(b_{i}(t), t\right) b_{i}^{\prime}(t)+\varphi\left(a_{i}(t), t\right) a_{i}^{\prime}(t) .
$$

Furthermore, for a given block $i$, we have $b_{i}^{\prime}(t)=a_{i}^{\prime}(t)=u_{i}$ and on the shock wave $\sigma^{\prime}=u_{s}$. Therefore, since $\varphi$ has a compact support $\Omega$, we compute

$$
\begin{aligned}
& A_{1}= \int_{a_{i+1}\left(t^{* *}\right)}^{\sigma\left(t^{* *}\right)} n_{i+1}^{*} S\left(u_{i+1}, \bar{p}_{i+1}\right) \varphi\left(x, t^{* *}\right) d x \\
&+\int_{\sigma\left(t^{*}\right)}^{b_{i+1}\left(t^{*}\right)} n_{i+1}^{*} S\left(u_{i+1}, \bar{p}_{i+1}\right) \varphi\left(x, t^{*}\right) d x \\
&+\int_{t^{*}}^{t^{* *}} n_{i+1}^{*} S\left(u_{i+1}, \bar{p}_{i+1}\right)\left(u_{i+1}-u_{s}\right) \varphi(\sigma(t), t) d t ; \\
& A_{2}=0 ; \\
& A_{3}=\int_{a_{i}\left(t^{*}\right)}^{\sigma\left(t^{*}\right)} n_{i}^{*} S\left(u_{i}, \tilde{p}_{i+1}\right) \varphi\left(x, t^{*}\right) d x \\
& \quad+\int_{\sigma\left(t^{* *}\right)}^{\tilde{a}_{i}\left(t^{* *}\right)} n_{i}^{*} S\left(u_{i}, \tilde{p}_{i+1}\right) \varphi\left(x, t^{* *}\right) d x \\
& \quad+\int_{t^{*}}^{t^{* *}} n_{i}^{*} S\left(u_{i}, \tilde{p}_{i+1}\right)\left(u_{s}-u_{i}\right) \varphi(\sigma(t), t) d t .
\end{aligned}
$$


Now

$$
a_{i}\left(t^{*}\right)=b_{i+1}\left(t^{*}\right)=\sigma\left(t^{*}\right), \sigma\left(t^{* *}\right)=\tilde{a}_{i}\left(t^{* *}\right), a_{i+1}\left(t^{* *}\right)=\sigma\left(t^{* *}\right),
$$

then

$$
\begin{aligned}
& <\partial_{t}(n S(u, \bar{p}))+\partial_{x}(n u S(u, \bar{p})), \varphi> \\
= & {\left[n_{i}^{*}\left(u_{s}-u_{i}\right) S\left(u_{i}, \tilde{p}_{i+1}\right)-n_{i+1}^{*}\left(u_{s}-u_{i+1}\right) S\left(u_{i+1}, \bar{p}_{i+1}\right)\right] \int_{t^{*}}^{t^{* *}} \varphi(\sigma(t), t) d t . }
\end{aligned}
$$

For $S(u, \bar{p})=1,(3.17)$ turns to

$$
\begin{aligned}
& <\partial_{t} n, \varphi>+<\partial_{x}(n u), \varphi> \\
= & {\left[n_{i}^{*}\left(u_{s}-u_{i}\right)-n_{i+1}^{*}\left(u_{s}-u_{i+1}\right)\right] \int_{t^{*}}^{t^{* *}} \varphi(\sigma(t), t) d t . }
\end{aligned}
$$

From (3.5), we have

$$
n_{i}^{*}\left(u_{i}-u_{s}\right)=n_{i+1}^{*}\left(u_{i+1}-u_{s}\right),
$$

therefore

$$
<\partial_{t} n, \varphi>+<\partial_{x}(n u), \varphi>=0 .
$$

For $S(u, \bar{p})=u+\bar{p}$, we obtain:

$$
\begin{aligned}
& <\partial_{t}(n(u+\bar{p})), \varphi>+<\partial_{x}(n(u+\bar{p}) u), \varphi> \\
= & {\left[\left(u_{i}+\tilde{p}_{i+1}\right)-\left(u_{i+1}+\bar{p}_{i+1}\right)\right] \times n_{i}^{*}\left(u_{s}-u_{i}\right) \int_{t^{*}}^{t^{* *}} \varphi(\sigma(t), t) d t, }
\end{aligned}
$$

which implies, thanks to (3.5) and (3.9)

$$
<\partial_{t}(n(u+\bar{p})), \varphi>+<\partial_{x}(n(u+\bar{p}) u)>=0 .
$$

Proposition 3.1. We have the maximum principle

$$
\underset{y}{\operatorname{essinf}} u^{0}(y) \leq u(x, t) \leq \underset{y}{\operatorname{esssup}} u^{0}(y)
$$

where esssup and essinf denote respectively the essential sup and the essential inf. We also have the bound

$$
0 \leq \bar{p}(x, t) \leq \underset{y}{\operatorname{esssup}} u^{0}(y)+\underset{y}{\operatorname{esssup}} \bar{p}^{0}(y) .
$$

Assume furthermore that the initial data in the blocks $u_{i}^{0}$ and $\bar{p}_{i}^{0}$ are $B V$ functions. Then we have for all $t \in[0, T]$

$$
\begin{gathered}
T V_{K}(u(., t)) \leq T V_{\tilde{K}}\left(u^{0}\right), \\
T V_{K}(\bar{p}(., t)) \leq T V_{\tilde{K}}\left(\bar{p}^{0}\right)+2 T V_{\tilde{K}}\left(u^{0}\right),
\end{gathered}
$$

for any compact $K=[a, b]$ and with $\tilde{K}=\left[a-t \operatorname{esssup}\left|u^{0}\right|, b-t \operatorname{essinf}\left|u^{0}\right|\right]$ where $T V_{K}$ (resp. $T V_{\tilde{K}}$ ) denotes the total variation on the set $K$ (resp. $\left.\tilde{K}\right)$. 
Proof. We gave below the proof of (3.26) for some extreme cases but the proof is general. For all $i=1 \ldots N$, when the block $i+1$ collides with the block $i$ then, $\bar{p}_{i+1}$ becomes $\tilde{p}_{i+1}=\bar{p}_{i+1}+u_{i+1}-u_{i}$. We assume the following dynamics: in a time interval $[0, t] \subset[0, T], j$ blocks collide successively at $t_{1}, \cdots, t_{j-1} \leq t$ for instance and then $N-j+1$ blocks collide at the same time $t_{s} \leq t$, then we have

$$
\begin{aligned}
T V_{K}(\bar{p}(t, .)) & =\left|\bar{p}_{1}^{0}-\tilde{p}_{2}^{t_{1}}\right|+\left|\tilde{p}_{2}^{t_{1}}-\tilde{p}_{3}^{t_{2}}\right|+\ldots+\left|\tilde{p}_{j-1}^{t_{j-2}}-\tilde{p}_{j}^{t_{j-1}}\right| \\
& +\left|\tilde{p}_{j}^{t_{s}}-\tilde{p}_{j+1}^{t_{s}}\right|+\ldots+\left|\tilde{p}_{N-1}^{t_{s}}-\tilde{p}_{N}^{t_{s}}\right| \\
& \leq\left|\bar{p}_{1}^{0}-\bar{p}_{2}^{0}\right|+\left|\bar{p}_{2}^{0}-\bar{p}_{3}^{0}\right|+\ldots+\left|\bar{p}_{j-1}^{0}-\bar{p}_{j}^{0}\right|+ \\
& 2\left(\left|u_{1}^{0}-u_{2}^{0}\right|+\left|u_{2}^{0}-u_{3}^{0}\right|+\ldots+\left|u_{j-1}^{0}-u_{j}^{0}\right|\right) \\
& +\left|\bar{p}_{j}^{t_{s-1}}-\bar{p}_{j+1}^{t_{s-1}}\right|+\ldots+\left|\bar{p}_{N-1}^{t_{s-1}}-\bar{p}_{N}^{t_{s-1}}\right|+ \\
& 2\left(\left|u_{j}^{t_{s-1}}-u_{j+1}^{t_{s-1}}\right|+\ldots+\left|u_{N-1}^{t_{s-1}}-u_{N}^{t_{s-1}}\right|\right) \\
& \leq T V_{\tilde{K}}\left(\bar{p}^{0}\right)+2 T V_{\tilde{K}}\left(u^{0}\right) .
\end{aligned}
$$

\subsection{Existence of a weak solution}

In the previous section we have proved the existence of solution to (3.1a)-(3.1c) for some particular data. We prove now that these particular initial data are dense, in some sense, in the set of desired initial data.

Lemma 3.1. Let $n^{0} \in L^{1}(\mathbb{R})$, $u^{0} \in L^{\infty}(\mathbb{R}) \cap B V(\mathbb{R})$ such that $0 \leq n^{0} \leq$ $n^{*}\left(u^{0}\right)$, then there exists a sequence of block initial data $\left(n_{k}^{0}, u_{k}^{0}, 0\right)_{k \geq 0}$ such that $\int_{\mathbb{R}} n_{k}^{0}(x) d x \leq \int_{\mathbb{R}} n^{0}(x) d x, \operatorname{essinf} u^{0} \leq u_{k}^{0} \leq \operatorname{esssup} u^{0}$ and $T V\left(u_{k}^{0}\right) \leq T V\left(u^{0}\right)$ for which the convergences $n_{k}^{0} \underset{k \rightarrow \infty}{\rightarrow} n^{0}$ and $n_{k}^{0} u_{k}^{0} \underset{k \rightarrow \infty}{\rightarrow} n^{0} u^{0}$ hold in the distribution sense.

Proof. The proof is widely inspired from the one of Lemma 4.1 in [3]. Let $k \in \mathbb{N}^{*}$ and let set $\forall i \in \mathbb{Z}$

$$
m_{i k}=\int_{\frac{i}{k}}^{\frac{i+1}{k}-\frac{1}{k^{2}}} n^{0}(x) d x .
$$

If $m_{i k} \neq 0$, we set

$$
\begin{aligned}
u_{i k}^{0} & =\inf _{\left[\frac{i}{k}, \frac{i+1}{k}\right]} u^{0}(x) d x, \\
n_{i k}^{0} & =n^{*}\left(u_{i k}^{0}\right) .
\end{aligned}
$$


We note that since $n^{0}(x) \leq n^{*}\left(u^{0}(x)\right) \leq n^{*}\left(u_{i k}^{0}\right)=n_{i k}^{0}$, then we have $\frac{m_{i k}}{n_{i k}^{0}}<\frac{1}{k}$. We finaly set, for any $x \in \mathbb{R}$,

$$
\begin{aligned}
& n_{k}^{0}(x)=\sum_{i=-k^{2}}^{k^{2}} n_{i k}^{0} \mathbb{I}_{] \frac{i}{k}, \frac{i}{k}+\frac{m_{i k}}{n_{i k}^{0}}}(x), \\
& \left.n_{k}^{0}(x) u_{k}^{0}(x)=\sum_{i=-k^{2}}^{k^{2}} n_{i k}^{0} u_{i k}^{0} \mathbb{I}\right]_{\frac{i}{k}, \frac{i}{k}+\frac{m_{i k}}{n_{i k}^{0}}}(x) .
\end{aligned}
$$

We extend the definition of $u_{k}^{0}$ in the vacuum as in Remark 3.1. We notice that we have

$$
T V_{[a, b]}\left(u_{k}^{0}\right) \leq T V_{[a-1 / k, b+1 / k]}\left(u^{0}\right) .
$$

Let $\varphi \in \mathcal{D}(\mathbb{R})$ and let $k_{0} \in \mathbb{N}$ such that $\operatorname{supp} \varphi \subset\left[-k_{0}, k_{0}\right]$. We have

$$
\begin{aligned}
\int_{\mathbb{R}} n_{k}^{0}(x) \varphi(x) d x & =\sum_{i=-k^{2}}^{k^{2}} \int_{\frac{i}{k}}^{\frac{i}{k}+\frac{m_{i k}}{n_{i k}}} n_{i k}^{0} \varphi(x) d x \\
& =\sum_{i=-k^{2}}^{k^{2}}\left[\varphi\left(\frac{i}{k}\right) m_{i k}+\varphi^{\prime}\left(x_{k}^{i}\right) \frac{m_{i k}^{2}}{2 n_{i k}^{0}}\right] \\
& =\sum_{i=-k^{2}}^{k^{2}}\left[\int_{\frac{i}{k}}^{\frac{i+1}{k}-\frac{1}{k^{2}}} n^{0}(x) \varphi\left(\frac{i}{k}\right) d x+\varphi^{\prime}\left(x_{k}^{i}\right) \frac{m_{i, k}^{2}}{2 n_{i k}^{0}}\right]
\end{aligned}
$$

where $x_{k}^{i} \in\left[\frac{i}{k}, \frac{i}{k}+\frac{m_{i k}}{n_{i k}^{0}}\right]$.

For $k>k_{0}$, we have

$$
\begin{aligned}
& \left|\int_{\mathbb{R}} n_{k}^{0}(x) \varphi(x) d x-\int_{\mathbb{R}} n^{0}(x) \varphi(x) d x\right| \\
\leq & \sum_{i=-k k_{0}}^{k k_{0}-1} \int_{\frac{i}{k}}^{\frac{i+1}{k}-\frac{1}{k^{2}}}\left\|n^{0}\right\|_{\infty}\left|\varphi\left(\frac{i}{k}\right)-\varphi(x)\right| d x+\sum_{i=-k k_{0}}^{k k_{0}-1} \int_{\frac{i+1}{k}-\frac{1}{k^{2}}}^{\frac{i+1}{k}}\left\|n^{0}\right\|_{\infty}|\varphi(x)| d x \\
& +\sum_{i=-k k_{0}}^{k k_{0}-1}\left|\varphi^{\prime}\left(x_{k}^{i}\right)\right| \frac{m_{i, k}^{2}}{2 n_{i k}^{0}} \\
\leq & \left\|n^{0}\right\|_{\infty}\left\|\varphi^{\prime}\right\|_{\infty} \sum_{i=-k k_{0}}^{k k_{0}-1} \int_{\frac{i}{k}}^{\frac{i+1}{k}-\frac{1}{k^{2}}}\left(x-\frac{i}{k}\right) d x+\left\|n^{0}\right\|_{\infty}\|\varphi\|_{\infty} \sum_{i=-k k_{0}}^{k k_{0}-1} \int_{\frac{i+1}{k}-\frac{1}{k^{2}}}^{\frac{i+1}{k}} d x \\
& +\left\|\varphi^{\prime}\right\|_{\infty} \sum_{i=-k k_{0}}^{k k_{0}-1}\left\|n^{0}\right\|_{\infty}\left(\int_{\frac{i}{k}}^{\frac{i+1}{k}-\frac{1}{k^{2}}} d x\right)^{2} \\
\leq & \frac{k_{0}}{k}\left\|n^{0}\right\|_{\infty}\left\|\varphi^{\prime}\right\|_{\infty}+\frac{2 k_{0}}{k}\left\|n^{0}\right\|_{\infty}\|\varphi\|_{\infty}+\frac{2 k_{0}}{k}\left\|n^{0}\right\|_{\infty}\left\|\varphi^{\prime}\right\|_{\infty}=O\left(\frac{1}{k}\right)
\end{aligned}
$$

and then

$$
\left\langle n_{k}^{0}, \varphi\right\rangle \underset{k \longrightarrow \infty}{\longrightarrow}\left\langle n^{0}, \varphi\right\rangle .
$$


Similarly we have

$$
\int_{\mathbb{R}} n_{k}^{0}(x) u_{k}^{0}(x) \varphi(x) d x=\sum_{-k^{2}}^{k^{2}}\left[\int_{\frac{i}{k}}^{\frac{i+1}{k}-\frac{1}{k^{2}}} n^{0}(x) u_{i k}^{0} \varphi\left(\frac{i}{k}\right) d x+u_{i k}^{0} \varphi^{\prime}\left(x_{k}^{i}\right) \frac{m_{i k}^{2}}{2 n_{i k}^{0}}\right],
$$

and the main difference to prove that

$$
\left\langle n_{k}^{0} u_{k}^{0}, \varphi\right\rangle \underset{k \longrightarrow \infty}{\longrightarrow}\left\langle n^{0} u^{0}, \varphi\right\rangle,
$$

is to show that

$$
A=\sum_{i=-k k_{0}}^{k k_{0}-1} \int_{\frac{i}{k}}^{\frac{i+1}{k}-\frac{1}{k^{2}}}\left|n^{0}(x)\right|\left|u_{i k}^{0}-u^{0}(x)\right|\left|\varphi\left(\frac{i}{k}\right)\right| d x \underset{k \longrightarrow \infty}{\longrightarrow} 0 .
$$

This last fact comes from the majoration

$$
\begin{aligned}
A & \leq \sum_{i=-k k_{0}}^{k k_{0}-1} \int_{\frac{i}{k}}^{\frac{i+1}{k}-\frac{1}{k^{2}}}\left\|n^{0}\right\|_{\infty}\|\varphi\|_{\infty}\left|u_{i k}^{0}-u^{0}(x)\right| d x \\
& \leq\left\|n^{0}\right\|_{\infty}\|\varphi\|_{\infty} \sum_{i=-k k_{0}}^{k k_{0}-1} \int_{\frac{i}{k}}^{\frac{i+1}{k}-\frac{1}{k^{2}}}\left|\sup _{\left[\frac{i}{k}, \frac{i+1}{k}\right]} u^{0}-\inf _{\left[\frac{i}{k}, \frac{i+1}{k}\right]} u^{0}\right| d x \\
& \leq \frac{1}{k}\left\|n^{0}\right\|_{\infty}\|\varphi\|_{\infty} \sum_{i=-k k_{0}}^{k k_{0}-1} T V_{x}\left(u^{0} ;\left[\frac{i}{k}, \frac{i+1}{k}\right]\right) \\
& \leq \frac{1}{k}\left\|n^{0}\right\|_{\infty}\|\varphi\|_{\infty} T V_{x}\left(u^{0} ;\left[-k_{0}, k_{0}\right]\right) .
\end{aligned}
$$

In this paper, due to the finite wave speed, we have $u_{k} \longrightarrow u$ in $L^{1}$ (we will come back to this assertion in the proof of the Theorem 3.2 below), therefore the passage to the limit is easier than in [5] where the technical Lemma 3.2 of [3] was required since we have not this strong convergence.

Lemma 3.2. [3] Let us assume that $\left(\gamma_{k}\right)_{k \in \mathbb{N}}$ is a bounded sequence in $L^{\infty}(\mathbb{R} \times] 0, T[)$ that tends to $\gamma$ in $L_{w^{*}}^{\infty}(\mathbb{R} \times] 0, \infty[)$, and satisfies for any $\Gamma \in$ $C_{c}^{\infty}(\mathbb{R})$,

$$
\int_{\mathbb{R}}\left(\gamma_{k}-\gamma\right)(x, t) \Gamma(x) d x \underset{k \longrightarrow \infty}{\longrightarrow} 0 \text {, in } L_{t}^{1}(] 0, T[) .
$$

Let us also assume that $\left(\omega_{k}\right)_{k \in \mathbb{N}}$ is a bounded sequence in $L^{\infty}(\mathbb{R} \times] 0, T[)$. If $\omega_{k} \longrightarrow \omega$ in $L^{1}$, then $\gamma_{k} \omega_{k} \rightarrow \gamma \omega$ in $L_{w^{*}}^{\infty}(\mathbb{R} \times] 0, \infty[)$, as $k \longrightarrow \infty$

We are searching solutions with the following regularities

$$
\begin{aligned}
& n \in L_{t}^{\infty}(] 0, \infty\left[, L_{x}^{\infty}(\mathbb{R}) \cap L_{x}^{1}(\mathbb{R})\right), \\
& u, \bar{p} \in L_{t}^{\infty}(] 0, \infty\left[, L_{x}^{\infty}(\mathbb{R})\right), \\
& 0 \leq n \leq n^{*}(u), \quad \bar{p}\left(n^{*}(u)-n\right)=0 .
\end{aligned}
$$

and the existence result is: 
Theorem 3.2. Let $\left(n^{0}, u^{0}, 0\right)$ be some initial data such that

$$
\begin{aligned}
& n^{0} \in L^{1}(\mathbb{R}) \cap L^{\infty}(\mathbb{R}), \\
& u^{0} \in L^{\infty}(\mathbb{R}) \cap B V(\mathbb{R}), \\
& \text { with } 0 \leq n^{0} \leq n^{*}\left(u^{0}\right) .
\end{aligned}
$$

Then there exists $(n, u, \bar{p})$ with regularities (3.33)-(3.35), solution to the system (3.1a)-(3.1c) with initial data $\left(n^{0}, u^{0}, 0\right)$. Moreover, this solution satisfies

$$
\begin{aligned}
& \underset{y}{\operatorname{essinf}} u^{0}(y) \leq u(x, t) \leq \underset{y}{\operatorname{esssup}} u^{0}(y) \\
& 0 \leq \bar{p}(x, t) \leq \underset{y}{\operatorname{esssup}} u^{0}(y)
\end{aligned}
$$

Proof. Let $n_{k}^{0}, u_{k}^{0}$ and $\bar{p}_{k}^{0}=0(k \in \mathbb{N})$ be the blocks initial data associated respectively to $n^{0}, u^{0}$ and $p^{0}=0$ provided by Lemma 3.1. For all $k$, the results of Section 3.2 allow us to get $\left(n_{k}, u_{k}, \bar{p}_{k}\right)$ solution of (3.1a)-(3.1c) with initial data $\left(n_{k}^{0}, u_{k}^{0}, p_{k}^{0}\right)$, satisfying (3.33)-(3.35). We are going to use the compactness result in Lemma 3.2 to prove that up to a subsequence, as $k \longrightarrow \infty,\left(n_{k}, u_{k}, \bar{p}_{k}\right) \rightarrow$ $(n, u, \bar{p})$ where $(n, u, \bar{p})$, with regularities (3.33)-(3.35), is a solution to (3.1a)(3.1c) for initial data $\left(n^{0}, u^{0}, \bar{p}^{0}\right)$.

Since $\left(n_{k}\right)$ is bounded in $L^{\infty}$, then there exists a subsequence such that

$$
n_{k} \rightarrow n \text { in } L^{\infty}(\mathbb{R} \times] 0, \infty[) w *
$$

Thanks to (3.23) and the bounds on $u_{k}^{0}$ provided by Lemma 3.1, the sequence $\left(u_{k}\right)_{k \geq 0}$ is uniformely bounded in $L^{\infty}(\mathbb{R} \times] 0, \infty[)$ and similarly $\left(\bar{p}_{k}\right)$ is bounded in $L^{\infty}$, then we can extract subsequences such that we also have

$$
u_{k} \rightarrow u \text { in } L^{\infty}(\mathbb{R} \times] 0, \infty[) w *, \quad \bar{p}_{k} \rightarrow \bar{p} \text { in } L^{\infty}(\mathbb{R} \times] 0, \infty[) w * .
$$

We want now to prove the passage to the limit in the equation.

First, we study an important new property of the model which is directly related to the finite speed of propagation and gives a strong compactness for the velocity. In order to get it, we study the variation with respect to $t$ of the $L_{x}^{1}$ norm of $u_{k}$. From the figure (2), we see that the worst case of evolution of this quantity is related to the computation of an area which is bounded by $\left\|u_{k}\right\|_{\infty}\left|t_{2}-t_{1}\right|$ times the variation of $u_{k}$ between two blocks. According to the definition of $u_{k}$ on the vacuum, the sum of all this quantity makes appear $T V\left(u^{0}\right)$.

Finally, we get that

$$
\int_{\mathbb{R}}\left|u_{k}\left(x, t_{2}\right)-u_{k}\left(x, t_{1}\right)\right| d x \leq\left\|u_{k}\right\|_{\infty}\left|t_{2}-t_{1}\right| T V\left(u^{0}\right)=C\left|t_{2}-t_{1}\right| .
$$

From the $B V_{x}$ bound on $u_{k}$ from (3.25), this equicontinuity with respect to $t$ and a Cantor diagonal process argument implies

$$
u_{k} \underset{k \rightarrow \infty}{\longrightarrow} u \text { in } L^{1}(\mathbb{R} \times[0, T]) .
$$

Similarly we also have

$$
\bar{p}_{k} \underset{k \rightarrow \infty}{\longrightarrow} \bar{p} \text { in } L^{1}(\mathbb{R} \times[0, T]) .
$$




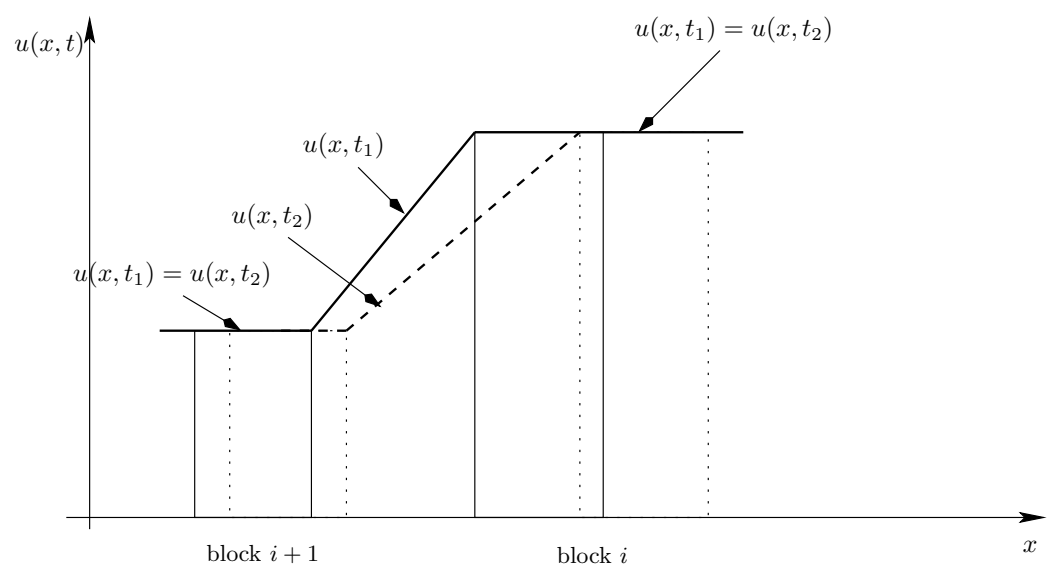

Figure 2: The $L^{1}$ equicontinuity with respect to $t$.

From the mass conservation equation, for any $\varphi \in C_{c}^{\infty}(\mathbb{R})$, the sequence $\int_{\mathbb{R}} n_{k}(t, x) \varphi(x) d x$ is bounded in $B V_{t}$. Then by Lemma 3.2, we have

$$
n_{k} u_{k} \underset{k \rightarrow \infty}{\rightarrow} n u \text { in } L_{w^{*}}^{\infty}(\mathbb{R} \times] 0, \infty[)
$$

Similarly, we get the convergences

$$
n_{k} \bar{p}_{k} \underset{k \rightarrow \infty}{\rightarrow} n \bar{p} \text { in } L_{w^{*}}^{\infty}(\mathbb{R} \times] 0, \infty[) .
$$

and

$$
n_{k}\left(u_{k}+\bar{p}_{k}\right) u_{k} \underset{k \rightarrow \infty}{\rightarrow} q u \text { in } L_{w^{*}}^{\infty}(\mathbb{R} \times] 0, \infty[),
$$

with $q=n u+n \bar{p}$.

To complete the proof, we are going to show that $n^{*}\left(u_{k}\right) \bar{p}_{k} \underset{k \rightarrow \infty}{\longrightarrow} n^{*}(u) \bar{p}$ in $L^{1}(\mathbb{R} \times[0, T])$. From (3.44) and (3.45), we have

$$
u_{k}(x, t) \underset{k \rightarrow \infty}{\longrightarrow} u(x, t), \bar{p}_{k}(x, t) \underset{k \rightarrow \infty}{\longrightarrow} \bar{p}(x, t) \quad \text { a.e. }(x, t) \in \mathbb{R} \times[0, T] .
$$

and there exists $h \in L^{1}(\mathbb{R} \times[0, T])$ such that for a subsequence $\left|\bar{p}_{k}\right| \leq h$ a.e. Since $n^{*}$ is continuous, we get

$$
n^{*}\left(u_{k}\right) \bar{p}_{k} \underset{k \rightarrow \infty}{\longrightarrow} n^{*}(u) \bar{p} \text { a.e., and }\left|n^{*}\left(u_{k}\right) \bar{p}_{k}\right| \leq n^{*}(0) h \in L^{1}(\mathbb{R} \times[0, T]),
$$

then by dominated convergence

$$
n^{*}\left(u_{k}\right) \bar{p}_{k} \underset{k \rightarrow \infty}{\longrightarrow} n^{*}(u) \bar{p} \text { in } L^{1}(\mathbb{R} \times[0, T]) .
$$

Finally we get a solution of (3.1a)-(3.1c). Moreover, this solution satisfies also (3.39)-(3.40).

Remark 3.2. We study here only the case where $p^{0}=0$. For the particular case of block initial data, we can nevertheless take any $p^{0}$ as in the corresponding section. The result can be extended to initial data such that on any interval $n=n^{*}$, the initial pressure is piecewise constant with 0 for the last constant, and 0 on other sets. 


\section{The Riemann Problem analysis}

\subsection{The Riemann problem for the RMAR* model (2.31)- (2.32)}

In this section, we briefly study the simple waves and then discuss the Riemann problem for the RMAR* system (2.31)-(2.32). And we refer the reader to [1] for more details on the derivation.

\subsubsection{Simple waves for the RMAR* system (2.31)-(2.32)}

Since the system (2.31)-(2.32) inherits the properties of the Modified $\mathrm{AR}^{*}$ model (2.23)-(2.24), the solution of the RMAR* model (2.31)-(2.32) consists of either a wave of the first family (1-shock or 1-rarefaction) or a wave of the second family (2-contact discontinuity). In this paragraph, we analyse the properties of these two families of waves.

First characteristic field. We obtain the wave of the first family when a left state $U_{l}^{\varepsilon}=\left(n_{l}^{\varepsilon}, u_{l}^{\varepsilon}\right)$ is connected with a right state $U_{r}^{\varepsilon}=\left(n_{r}^{\varepsilon}, u_{r}^{\varepsilon}\right)$ through the curve

$$
u_{r}^{\varepsilon}+\varepsilon p\left(n_{r}^{\varepsilon}, u_{r}^{\varepsilon}\right)=u_{l}^{\varepsilon}+\varepsilon p\left(n_{l}^{\varepsilon}, u_{l}^{\varepsilon}\right) .
$$

- If $u_{r}^{\varepsilon}<u_{l}^{\varepsilon}$, this wave (of the first family) is a 1-shock i.e. a jump discontinuity, travelling with the speed

$$
\sigma^{\varepsilon}=\frac{n_{r}^{\varepsilon} u_{r}^{\varepsilon}-n_{l}^{\varepsilon} u_{l}^{\varepsilon}}{n_{r}^{\varepsilon}-n_{l}^{\varepsilon}} .
$$

- On the other hand, if $u_{r}^{\varepsilon}>u_{l}^{\varepsilon}$, this wave of the first family is a 1-rarefaction i.e. a continuous solution of the form $\left(n^{\varepsilon}, u^{\varepsilon}\right)(\xi)$ (with $\xi=\frac{x}{t}$ ) given by

$$
\begin{array}{r}
\left(\begin{array}{c}
\left(n^{\varepsilon}\right)^{\prime}(\xi) \\
\left(u^{\varepsilon}\right)^{\prime}(\xi)
\end{array}\right)=\frac{r_{1}^{\varepsilon}\left(U^{\varepsilon}(\xi)\right)}{\nabla \lambda_{1}^{\varepsilon}\left(U^{\varepsilon}(\xi)\right) \cdot r_{1}^{\varepsilon}\left(U^{\varepsilon}(\xi)\right)}, \quad \lambda_{1}^{\varepsilon}\left(U_{l}^{\varepsilon}\right) \leq \xi \leq \lambda_{1}^{\varepsilon}\left(U_{r}^{\varepsilon}\right), \\
\left(n^{\varepsilon}, u^{\varepsilon}\right)(\xi)=\left\{\begin{array}{llc}
\left(n_{l}^{\varepsilon}, u_{l}^{\varepsilon}\right) & \text { for } & \xi<\lambda_{1}^{\varepsilon}\left(U_{l}^{\varepsilon}\right), \\
\left(n_{r}^{\varepsilon}, u_{r}^{\varepsilon}\right) & \text { for } & \xi>\lambda_{2}^{\varepsilon}\left(U_{r}^{\varepsilon}\right) .
\end{array}\right.
\end{array}
$$

Second characteristic field. We obtain a wave of the second family i.e. a 2-contact discontinuity when $u_{l}^{\varepsilon}=u_{r}^{\varepsilon}$. In this case, this cantact discontinuity between the left state $U_{l}^{\varepsilon}=\left(n_{l}^{\varepsilon}, u_{r}^{\varepsilon}\right)$ and the right state $U_{r}^{\varepsilon}=\left(n_{r}^{\varepsilon}, u_{r}^{\varepsilon}\right)$ travels with speed $u^{\varepsilon}=u_{r}^{\varepsilon}=u_{l}^{\varepsilon}$.

\subsubsection{Solution to the Riemann problem for the RMAR* sys- tem (2.31)-(2.32)}

Let $U_{l}^{\varepsilon}=\left(n_{l}^{\varepsilon}, u_{l}^{\varepsilon}\right)$ and $U_{r}^{\varepsilon}=\left(n_{r}^{\varepsilon}, u_{r}^{\varepsilon}\right)$ be two given states respectively on the left and on the right. The general solution to the Riemann problem for the RMAR* model (2.31)-(2.32) consists of two simples waves separated by an intermediate state $\tilde{U}^{\varepsilon}=\left(\tilde{n}^{\varepsilon}, \tilde{u}^{\varepsilon}\right)$ which is the intersection point between the 1- wave curve 
through $U_{l}^{\varepsilon}$ and the 2-contact discontinuity through $U_{r}^{\varepsilon}$. First, $U_{l}^{\varepsilon}$ is connected with $\tilde{U}^{\varepsilon}$ through a wave of the first family (i.e. either 1-shock or 1-rarefaction according to the above discussion) and then $\tilde{U}^{\varepsilon}$ is connected with $U_{r}^{\varepsilon}$ through a 2-contact discontinuity. Therefore,

$$
\tilde{u}^{\varepsilon}+\varepsilon p\left(\tilde{n}^{\varepsilon}, \tilde{u}^{\varepsilon}\right)=u_{l}^{\varepsilon}+\varepsilon p\left(n_{l}^{\varepsilon}, u_{l}^{\varepsilon}\right) \quad \text { and } \quad \tilde{u}^{\varepsilon}=u_{r}^{\varepsilon} .
$$

Hence, the density of the intermediate state $\tilde{U}^{\varepsilon}=\left(\tilde{n}^{\varepsilon}, \tilde{u}^{\varepsilon}=u_{r}^{\varepsilon}\right)$ is given by

$$
\tilde{n}^{\varepsilon}=p\left(., u_{r}^{\varepsilon}\right)^{-1}\left[\varepsilon^{-1}\left(u_{l}^{\varepsilon}-u_{r}^{\varepsilon}\right)+p\left(n_{l}^{\varepsilon}, u_{l}^{\varepsilon}\right)\right] .
$$

Remark 4.1. If $u_{r}^{\varepsilon}>u_{l}^{\varepsilon}$, equation (4.6) admits a solution if and only if $\varepsilon^{-1}\left(u_{l}^{\varepsilon}-u_{r}^{\varepsilon}\right)+p\left(n_{l}^{\varepsilon}, u_{l}^{\varepsilon}\right)>0$. Otherwise i.e. if $u_{l}^{\varepsilon}+\varepsilon p\left(n_{l}^{\varepsilon}, u_{l}^{\varepsilon}\right)<u_{r}^{\varepsilon}$, then a vacuum state $(n=0)$ separates the two states $U_{l}^{\varepsilon}$ and $U_{r}^{\varepsilon}$.

Now let us discuss the solution to the Riemann problem in different cases.

(1) $u_{r}^{\varepsilon}<u_{l}^{\varepsilon}$. First, a 1-shock connects $U_{l}^{\varepsilon}=\left(n_{l}^{\varepsilon}, u_{l}^{\varepsilon}\right)$ to the intermediate state $\tilde{U}^{\varepsilon}=\left(\tilde{n}^{\varepsilon}, u_{r}^{\varepsilon}\right)$ and then a 2-contact discontinuity connects $\tilde{U}^{\varepsilon}$ to $U_{r}^{\varepsilon}=\left(n_{r}^{\varepsilon}, u_{r}^{\varepsilon}\right)$.

(2) $u_{l}^{\varepsilon}<u_{r}^{\varepsilon}<u_{l}^{\varepsilon}+\varepsilon p\left(n_{l}^{\varepsilon}, u_{l}^{\varepsilon}\right)$. The left state $U_{l}^{\varepsilon}=\left(n_{l}^{\varepsilon}, u_{l}^{\varepsilon}\right)$ is connected to the intermediate state $\tilde{U}^{\varepsilon}=\left(\tilde{n}^{\varepsilon}, u_{r}^{\varepsilon}\right)$ by a 1-rarefaction wave and then a 2-contact discontinuity connects $\tilde{U}^{\varepsilon}$ to the right state $U_{r}^{\varepsilon}=\left(n_{r}^{\varepsilon}, u_{r}^{\varepsilon}\right)$.

(3) $u_{l}^{\varepsilon}+\varepsilon p\left(n_{l}^{\varepsilon}, u_{l}^{\varepsilon}\right)<u_{r}^{\varepsilon}$. First a 1-rarefaction wave connects the left state $U_{l}^{\varepsilon}=\left(n_{l}^{\varepsilon}, u_{l}^{\varepsilon}\right)$ to the vacuum $\left(\left(0, \tilde{u}^{\varepsilon}\right)\right.$ with $\left.\tilde{u}^{\varepsilon}=u_{l}^{\varepsilon}+\varepsilon p\left(n_{l}^{\varepsilon}, u_{l}^{\varepsilon}\right)\right)$ and then a 2-contact discontinuity connects the vacuum to the right state $U_{r}^{\varepsilon}=\left(n_{r}^{\varepsilon}, u_{r}^{\varepsilon}\right)$.

\subsection{Simple waves for the SOMC system (2.34)-(2.36)}

We recall that the SOMC system (2.34)-(2.36) is the formal limit of the RMAR* system (2.31)-(2.32) when $\varepsilon \longrightarrow 0$. As for the CPGD system (2.19)-(2.21), due to $(2.36), \bar{p}$ plays the role of a Lagrangian multiplier. Therefore one has to distinguish between the cases $n=n^{*}(u)$ and $n<n^{*}(u)$. Throughout the discussion below we consider the three quantities $n, u$ and $\bar{p}$. Let $U_{l}=\left(n_{l}, u_{l}, \bar{p}_{l}\right)$ and $U_{r}=\left(n_{r}, u_{r}, \bar{p}_{r}\right)$ be two given states respectively on the left and on the right such that

$$
\left(n_{l}^{\varepsilon}, u_{l}^{\varepsilon}, \varepsilon p\left(n_{l}^{\varepsilon}, u_{l}^{\varepsilon}\right)\right) \underset{\varepsilon \longrightarrow 0}{\longrightarrow}\left(n_{l}, u_{l}, \bar{p}_{l}\right)
$$

and

$$
\left(n_{r}^{\varepsilon}, u_{r}^{\varepsilon}, \varepsilon p\left(n_{r}^{\varepsilon}, u_{r}^{\varepsilon}\right)\right) \underset{\varepsilon \longrightarrow 0}{\longrightarrow}\left(n_{r}, u_{r}, \bar{p}_{r}\right) .
$$

If $n_{l, r}=n^{*}\left(u_{l, r}\right)$, then $\lim _{\varepsilon \longrightarrow 0} \varepsilon p\left(n_{l, r}^{\varepsilon}, u_{l, r}^{\varepsilon}\right)=\bar{p}_{l, r}$ with $0 \leq \bar{p}_{l, r}<\infty$.

\subsubsection{First characteristic field: 1-shocks.}

The 1-shock waves appear in the SOMC system only if $u_{r}<u_{l}$ and if (4.5) is satisfied.

We shall distinguish the following cases. 
1. $n_{l}<n^{*}\left(u_{l}\right), n_{r}=n^{*}\left(u_{r}\right)$ i.e. $\bar{p}_{l}=0$ and $0<\bar{p}_{r}<\infty$.

In this case, when $\varepsilon \longrightarrow 0$, from (4.1) we have $u_{l}=u_{r}+\bar{p}_{r}>u_{r}$. Therefore, we have a 1-shock between the states $U_{l}=\left(n_{l}, u_{l}, \bar{p}_{l}=0\right)$ and $U_{r}=$ $\left(n^{*}\left(u_{r}\right), u_{r}, \bar{p}_{r}\right)$ travelling with a speed $\sigma$ given by

$$
\sigma=\frac{n^{*}\left(u_{r}\right) u_{r}-n_{l} u_{l}}{n^{*}\left(u_{r}\right)-n_{l}}
$$

This situation models a "cluster growing" upstream: as soon as a faster vehicle catches up the cluster, it adapts its velocity to the saturation density $n^{*}\left(u_{r}\right)$ and "is swallowed by" the cluster.

2. $n_{l}=n^{*}\left(u_{l}\right), n_{r}=n^{*}\left(u_{r}\right)$ i.e. $0<\bar{p}_{l}<\infty$ and $0<\bar{p}_{r}<\infty$.

When $\bar{p}_{r}>\bar{p}_{l}$, from (4.1), we have

$$
u_{l}+\bar{p}_{l}=u_{r}+\bar{p}_{r} \Longrightarrow u_{l}=u_{r}+\bar{p}_{r}-\bar{p}_{l}>u_{r} .
$$

Therefore we have a 1-shock travelling with the speed

$$
\sigma=\frac{n^{*}\left(u_{r}\right) u_{r}-n^{*}\left(u_{l}\right) u_{l}}{n^{*}\left(u_{r}\right)-n^{*}\left(u_{l}\right)}
$$

This situation models a "cluster slowing down" that leads to a merging of two clusters since the left cluster is faster than the right one. In contrast with the corresponding discussion in [5], here the wave speed $\sigma$ is always finite. As soon as the collusion occurs, the velocity of the left cluster adjusts gradually to the right one through the shock wave. This propagation also involves the functional $\bar{p}_{l}$, see below.

\subsubsection{First characteristic field: 1-rarefaction Waves.}

The 1-rarefaction waves appear in the SOMC system if $u_{r}>u_{l}$ and (4.5) is satisfied.

Here also we shall distinguish two cases.

1. $n_{l}=n^{*}\left(u_{l}\right), n_{r}<n^{*}\left(u_{r}\right)$, therfore $0<\bar{p}_{l}<\infty$ and $\bar{p}_{r}=0$.

When $\varepsilon \longrightarrow$, from (4.1) we have $u_{r}=u_{l}+\bar{p}_{l}>u_{l}$. This case describes a "cluster acceleration" leading to a "cluster growing" downstream. Indeed, the vehicles downstream are faster than the cluster: the cluster accelerates in order to reach its preferred velocity $u_{l}+\bar{p}_{l}=u_{r}$.

Here also, in contrast to [5], the velocity of the left state $u_{l}$ changes gradually to $u_{r}$, therefore the functional $\bar{p}_{l}$ changes also gradually to $\tilde{p}_{l} \longrightarrow 0$.

2. $n_{l}=n^{*}\left(u_{l}\right), n_{r}=n^{*}\left(u_{r}\right)$, therefore $0<\bar{p}_{l}<\infty$ and $0<\bar{p}_{r}<\infty$ When $\bar{p}_{l}>\bar{p}_{r}$, from (4.1) we have

$$
u_{r}+\bar{p}_{r}=u_{l}+\bar{p}_{l} \Longrightarrow u_{r}=u_{l}+\bar{p}_{l}-\bar{p}_{r}>u_{l} .
$$

This situation also models a "cluster acceleration" that leads to a merging of two clusters. The right cluster being faster than the left one, the left cluster accelerates and catches up the right one since $u_{r}=u_{l}+\bar{p}_{l}-\bar{p}_{r}<$ $u_{l}+\bar{p}_{l}$. Hence the left cluster adjusts gradually its velocity to the velocity $u_{r}$ of the right one. 


\subsubsection{Second characteristic field: 2-contact discontinuities}

The 2-contact discontinuities appear in the SOMC system if $u_{r}=u_{l}$. We have following cases.

1. $n_{l}<n^{*}\left(u_{l}\right), n_{r}<n^{*}\left(u_{r}\right)$, therefore $\bar{p}_{l}=\bar{p}_{r}=0$,

2. $n_{l}=n^{*}\left(u_{l}\right), n_{r}=n^{*}\left(u_{r}\right)$ with $0<\bar{p}_{l}=\bar{p}_{r}<\infty$.

In each of these two cases, at the limit $\varepsilon \longrightarrow 0$ in (4.1), we get $u_{l}=u_{r}=$ $\tilde{u}$. Therefore, the solution consists of a 2-contact discontinuity travelling with velociy $\tilde{u}$ from $U_{l}=\left(n_{l}, \tilde{u}, \bar{p}_{l}\right)$ to $U_{r}=\left(n_{r}, \tilde{u}, \bar{p}_{r}\right)$.

\subsection{Solution to the Riemann problem for the SOMC sys- tem (2.34)-(2.35)}

In this subsection, we describe the solutions to the Riemann problem for the SOMC system (2.34)-(2.35), by combining the previously described elementary waves depending on whether $n=n *(u)$ or $n<n^{*}(u)$.

Let $U_{l}=\left(n_{l}, u_{l}, \bar{p}_{l}\right)$ and $U_{r}=\left(n_{r}, u_{r}, \bar{p}_{r}\right)$ be the initial data on the left and on the right, respectively. The solutions to the SOMC system (2.34)-(2.36) system for these initial data consist of the following cases.

4.3.1 Case $1 n_{l}<n^{*}\left(u_{l}\right), n_{r}<n^{*}\left(u_{r}\right)$ i.e. $\bar{p}_{l}=\bar{p}_{r}=0$.

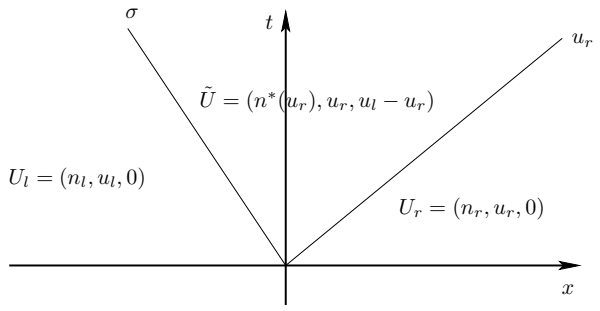

(a)

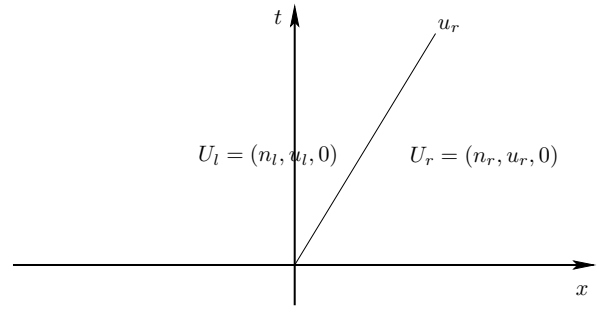

(b)

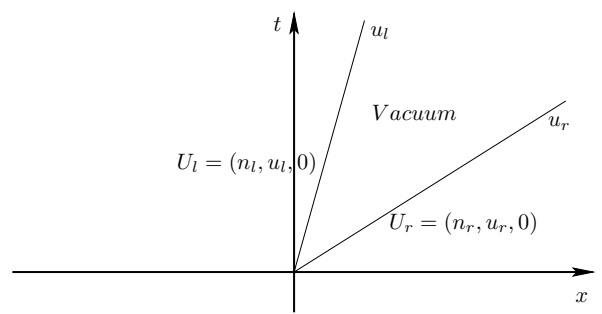

(c)

Figure 3: (a): Subcase 1.1, (b): Subcase 1.2, (c): Subcase 1.3 
Subcase $1.1 u_{r}<u_{l}$. In this case we have a cluster formation. In fact the density of the intermediate state $\tilde{n}$ increases and tends to $n^{*}\left(u_{r}\right)$. At the same time, $\varepsilon p\left(\tilde{n}, u_{r}\right) \longrightarrow \tilde{p}=u_{l}-u_{r}$. Therefore the intermediate state that characterizes the cluster is given by $\tilde{U}=\left(n^{*}\left(u_{r}\right), u_{r}, \tilde{p}=u_{l}-u_{r}\right)$. This intermediate is separated from the left state $U_{l}=\left(n_{l}, u_{l}, \bar{p}_{l}=0\right)$ and the right state $U_{r}=\left(n_{r}, u_{r}, \bar{p}_{r}=0\right)$ respectively by a 1 -shock travelling with velocity $\sigma$ and a contact discontinuity with velocity $u_{r}$. The schock speed $\sigma$ is given by

$$
\sigma=\frac{n_{r} u_{r}-n_{l} u_{l}}{n_{r}-n_{l}}
$$

This situation is illustrated by Figure 3 (a).

Subcase $1.2 u_{l}<u_{r}<u_{l}+p_{l}$, therefore $u_{l}=u_{r}$. This case is solved by a single contact discontinuity travelling with the velocity $\tilde{u}=u_{l}=u_{r}$ that connects $U_{l}=\left(n_{l}, u_{l}, \bar{p}_{l}=0\right)$ with $U_{r}=\left(n_{r}, u_{r}, \bar{p}_{r}=0\right)$. An example is shown in Figure $3(\mathrm{~b})$.

Subcase $1.3 u_{l}+p_{l}<u_{r}$ i.e. $u_{l}<u_{r}$. In this situation the vacuum appears. It is separated from the left state $U_{l}=\left(n_{l}, u_{l}, \bar{p}_{l}=0\right)$ by contact discontinuity (with velocity $u_{l}$ ) and another contact discontinuity (with velocity $u_{r}$ ) connects the vacuum with the right state $U_{r}=\left(n_{r}, u_{r}, \bar{p}_{r}=0\right)$, see Figure 3 (c) for illustration.

4.3.2 Case $2 n_{l}=n^{*}\left(u_{l}\right), n_{r}<n^{*}\left(u_{r}\right)$ i.e. $0 \leq \bar{p}_{l}<\infty$ and $\bar{p}_{r}=0$.

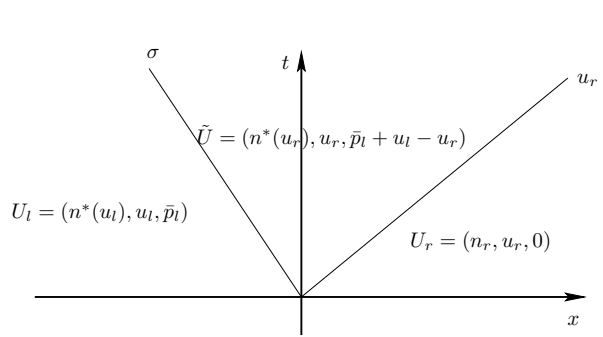

(a)

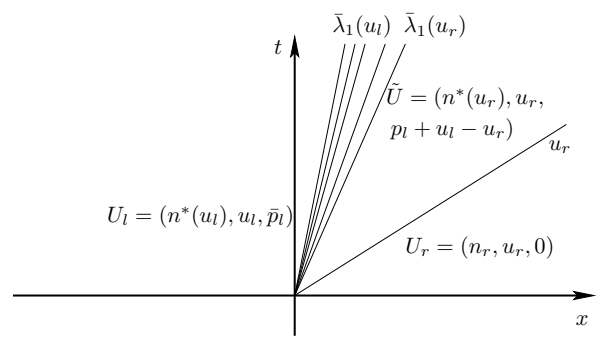

(b)

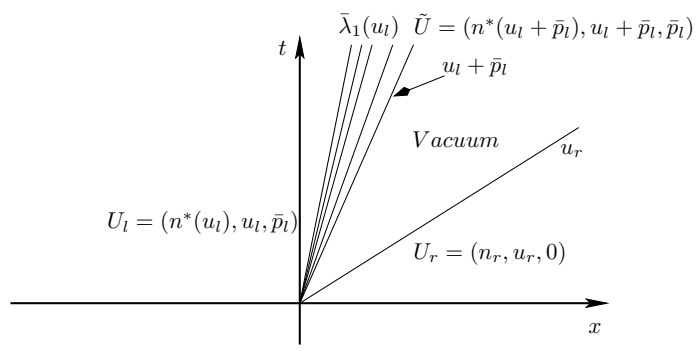

(c)

Figure 4: (a): Subcase 2.1, Subcase 2.2, (b): Subcase 2.3 
Subcase 2.1 $u_{r}<u_{l}$. Here we have a "cluster growing" downstream. The cluster being faster than the vehicles ahead, must adapt its velocity gradually to $u_{r}$ through a 1 -shock connecting $u_{r}$ to the intermediate state $\tilde{U}=$ $\left(n^{*}\left(u_{r}\right), u_{r}, \tilde{p}=\bar{p}_{l}+u_{l}-u_{r}\right)$. This is illustrated by Figure 4 (a).

Subcase 2.2 $u_{l}<u_{r}<u_{l}+\bar{p}_{l}$. The vehicles ahead of the cluster are faster than this one. However, their velocity $u_{r}$ is less than the cluster preferred velocity $u_{l}+\bar{p}_{l}$ therefore, we have a "cluster acceleration" that leads to a "cluster growing" downstream. The solution is of the following form: The left state $U_{l}=\left(n^{*}\left(u_{l}\right), u_{l}, \bar{p}_{l}\right)$ is connected to the intermediate state $\tilde{U}=\left(n^{*}\left(u_{r}\right), u_{r}, \tilde{p}=\right.$ $\left.\bar{p}_{l}+u_{l}-u_{r}\right)$ with a 1-rarefaction wave, then $\tilde{U}$ is connected to $U_{r}=\left(n_{r}, u_{r}, \bar{p}_{r}=\right.$ 0 ) with a contact discontinuity of velocity $u_{r}$. This is illustrated by (b) of Figure 4 .

Subcase 2.3: $u_{l}+\bar{p}_{l}<u_{r}$. Here the velocity $u_{r}$ of the vehicles ahead of the cluster is greater than the cluster preferred velocity $u_{l}+\bar{p}_{l}$. Therefore we have a "cluster acceleration" to reach the preferred velocity $u_{l}+\bar{p}_{l}$ but a vacuum appears since $u_{r}>u_{l}+\bar{p}_{l}$. The solution is as follows: The left state $U_{l}=$ $\left(n^{*}\left(u_{l}\right), u_{l}, \bar{p}_{l}\right)$ is connected to the intermediate state $\tilde{U}=\left(n^{*}\left(u_{l}+\bar{p}_{l}\right), u_{l}+\bar{p}_{l}, \bar{p}_{l}\right)$ through a 1-rarefaction wave. Then $\tilde{U}$ is connected to the vacuum by a 2 contact discontinuity. Then the vacuum is connected with $U_{r}$ by a 2 -contact discontinuity. An example is described in Figure 4 (c).

4.3.3 Case $3 n_{l}<n^{*}\left(u_{l}\right), n_{r}=n^{*}\left(u_{r}\right)$ i.e. $\bar{p}_{l}=0$ and $0 \leq \bar{p}_{r}<\infty$.

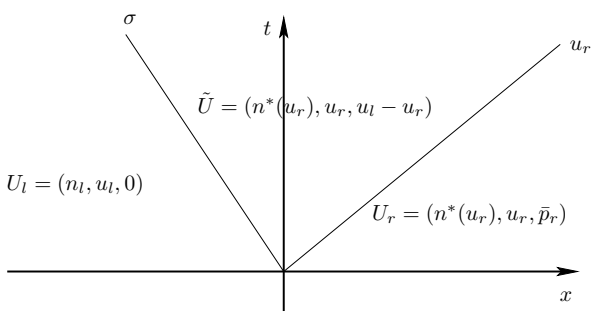

(a)

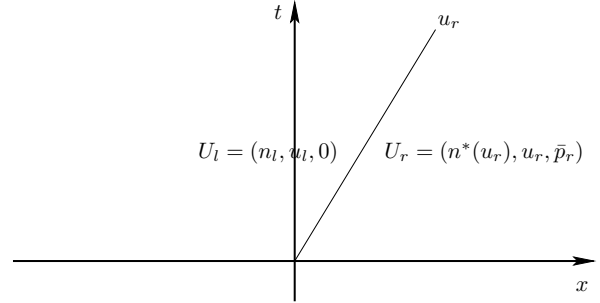

(b)

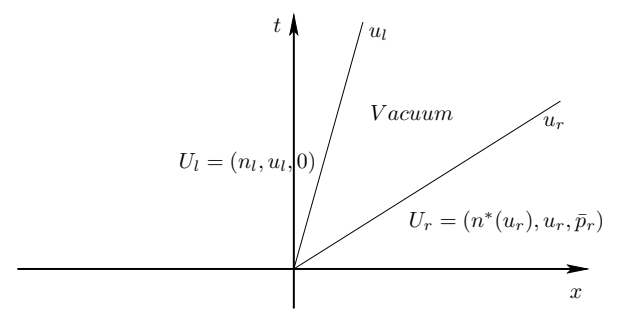

(c)

Figure 5: (a): Subcase 3.1, (b): Subcase 3.2, (c): Subcase 3.3 
Subcase 3.1 $u_{r}<u_{l}$. In this situation we have a "cluster growing" upstream. The vehicles behind the cluster are faster that this one. As soon as a vehicle catches up the cluster, it slows down, adapts its velocity to the saturation dentity $n^{*}\left(u_{r}\right)$ through a 1-shock connecting it to an intermediate $\tilde{U}$ and becomes a part of (or "is swallowed by") the cluster. The solution is quasi similar to that of Subcase 1.1 and the only difference is that here $p_{r} \neq 0$. See Figure 5 (a).

Subcase $3.2 u_{l}<u_{r}<u_{l}+\bar{p}_{l}=u_{l}$, therefore $u_{l}=u_{r}$. Like in the Subcase 1.2 , here also the solution consists of a single contact discontinuity with velocity $\tilde{u}=u_{l}=u_{r}$, connecting the left state $U_{l}=\left(n_{l}, u_{l}, \bar{p}_{l}=0\right)$ and the right state $U_{r}=\left(n^{*}\left(u_{r}\right), u_{r}, \bar{p}_{r}\right)$. See Figure $5(\mathrm{~b})$.

Subcase $3.3 u_{l}+\bar{p}_{l}<u_{r}$, therefore $u_{l}<u_{r}$. The downstream cluster being faster than the vehicles behind, a vacuum state appears between them. Since $\bar{p}_{l}=0$, the left state $U_{l}=\left(n_{l}, u_{l}, \bar{p} l=0\right)$ is connected to the vacuum with a contact discontinuity of velocity $u_{l}$. Then the vacuum is separated from the right state $U_{r}=\left(n^{*}\left(u_{r}\right), u_{r}, \bar{p}_{r}\right)$ with another contact discontinuity of velocity $u_{r}$. See (c) of Figure 5 for illustration.

4.3.4 Case $4 n_{l}=n^{*}\left(u_{l}\right), n_{r}=n^{*}\left(u_{r}\right)$ i.e. $0<p_{r}<\infty, 0<\bar{p}_{l}<\infty$.

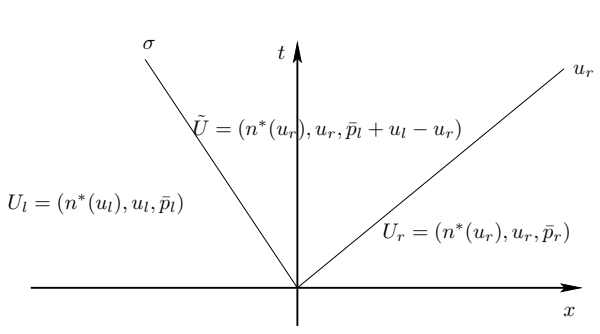

(a)

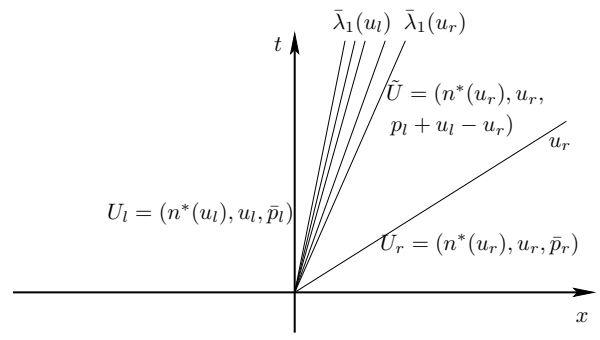

(b)

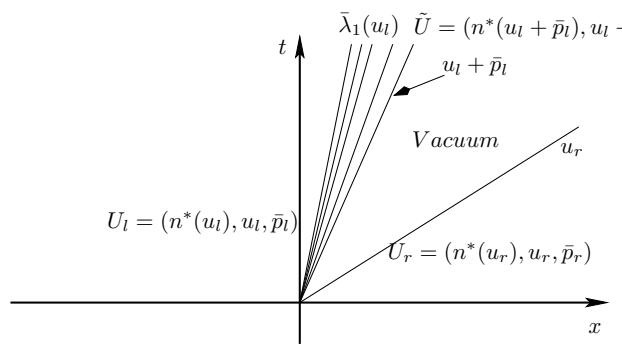

(c)

Figure 6: (a): Subcase 4.1, (b): Subcase 4.2, (c): Subcase 4.3

Subcase $4.1 u_{r}<u_{l}$. Here we have a "cluster slowing down", a 1-shock leading to a merging of two clusters. The left cluster is faster than the right one. When the two clusters meet, the left one slows down and adapts gradually its velocity to the velocity $u_{r}$ of the right one. The solution is therefore almost 
similar to the one of Subcase 1.1 except that here the intermediate state is now given by $\tilde{U}=\left(n^{*}\left(u_{r}\right), u_{r}, \tilde{p}=\bar{p}_{l}+u_{l}-u_{r}\right)$, with here $p_{l} \neq 0$ and $p_{r} \neq 0$. See Figure 6 (a) for illustration.

Subcase $4.2 u_{l}<u_{r}<u_{l}+p_{l}$. In this situation we have a "cluster acceleration" leading to the merging of two clusters. The right cluster is faster than the left one, but its velocity $u_{r}$ is less than the preferred velocity $u_{l}+\bar{p}_{l}$ of the left cluster, which then accelerates and gradually adapts its velocity to $u_{r}$. The solution is almost similar to the one of Subcase 2.2, except that here $p_{r} \neq 0$. An example is given by Figure 6 (b).

Subcase $4.3 u_{l}+\bar{p}_{l}<u_{r}$. The velocity $u_{r}$ of the right cluster is larger than the preferred velocity of the left cluster $u_{l}+\bar{p}_{l}$. Therefore the left cluster accelerates to reach its preferred velocity. However, the two clusters do not collide since $u_{r}>u_{l}+\bar{p}_{l}$, so that a vacuum state appears between them, as in Subcase 2.3 (with here $p_{r} \neq 0$ ). We have illustrated this situation in Figure 6 (c).

\section{Concluding remarks}

The model presented in this paper, contrarily to [5], takes into acount the fact that the maximal density depends on the velocity. Furthermore, the proposed model behaves as the Lighthill \& Whitham model [17] when the maximal density constraint is saturated, and on the other hand in the free flow regime, it becomes a pressureless gas model. This double-sided bihaviour has been highlighted in the analysis of the Riemann problem. We have proved an existence result of weak solution for the model and discussed the associated Riemann problem. This work is motivated by the fact that in practice a correlation exists between the maximal density constraint and the velocity. The approach in this paper opens many perspectives, and futur research can be carried out towards several directions. First, this model is designed on a single highway framework. A further interesting issue is to extend the model to the case of multilanes highways with overtaking possibilities. Also an extension to road networks and a comparison with other traffic models would be worthwhile.

\section{Appendix}

Proof of Lemma 2.1. We have

$$
\nabla \lambda_{1} \cdot r_{1}=-2 \partial_{n} p-n\left(\begin{array}{c}
1 \\
\frac{-\partial_{n} p}{1+\partial_{u} p}
\end{array}\right)^{t}\left(\begin{array}{cc}
\partial_{n n} p & \partial_{u n} p \\
\partial_{u n} p & \partial_{u u} p
\end{array}\right)\left(\begin{array}{c}
1 \\
\frac{-\partial_{n} p}{1+\partial_{u} p}
\end{array}\right)
$$

As $\partial_{n} p \geq 0$, the first term at the right hand side of the equation (5.1) is non positive.

Let us denote by $H(n, u)$ the Hessian matrix of $p\left(\begin{array}{cc}\partial_{n n} p & \partial_{u n} p \\ \partial_{u n} p & \partial_{u u} p\end{array}\right)$.

Rewriting, $H(n, u)$ in terms of $\left(n, n^{*}\right)$ and the derivative of $n^{*}$, we obtain, 


$$
\begin{aligned}
H(n, u) & =\left(\begin{array}{cc}
\partial_{n n} p^{\dagger} & \partial_{n n^{*}} p^{\dagger} \frac{d n^{*}(u)}{d u} \\
\partial_{n n^{*}} p^{\dagger} \frac{d n^{*}(u)}{d u} & \partial_{n^{*} n^{*}} p^{\dagger}\left(\frac{d n^{*}(u)}{d u}\right)^{2}+\partial_{n^{*}} p^{\dagger} \frac{d^{2} n^{*}(u)}{d u^{2}}
\end{array}\right) \\
& =\left(\begin{array}{cc}
1 & 0 \\
0 & \frac{d n^{*}(u)}{d u}
\end{array}\right)\left(\begin{array}{cc}
\partial_{n n} p^{\dagger} & \partial_{n n^{*}} p^{\dagger} \\
\partial_{n n^{*}} p^{\dagger} & \partial_{n^{*} n^{*}} p^{\dagger}
\end{array}\right)\left(\begin{array}{cc}
1 & 0 \\
0 & \frac{d n^{*}(u)}{d u}
\end{array}\right)+ \\
& \left(\begin{array}{cc}
0 & 0 \\
0 & \partial_{n^{*}} p^{\dagger} \frac{d^{2} n^{*}(u)}{d u^{2}}
\end{array}\right) .
\end{aligned}
$$

with

$$
p^{\dagger}\left(n, n^{*}\right)=\left(\frac{1}{n}-\frac{1}{n^{*}}\right)^{-\gamma} \text { and } \gamma>0 .
$$

Let us denote by $\tilde{H}\left(n, n^{*}\right)$ the matrix $\left(\begin{array}{cc}\partial_{n n} p^{\dagger} & \partial_{n n^{*}} p^{\dagger} \\ \partial_{n n^{*}} p^{\dagger} & \partial_{n^{*} n^{*}} p^{\dagger}\end{array}\right)$.

In order to show that $\nabla \lambda_{1} \cdot r_{1}$ keeps a constant sign $\left(\nabla \lambda_{1} \cdot r_{1}<0\right)$ we are looking for a condition such that $H\left(n, n^{*}\right)$ is, positive definite. Since $\forall \gamma>0$, we have

$$
\begin{aligned}
& \partial_{n} p^{\dagger}=\frac{\gamma}{n^{2} Z^{\gamma+1}}>0 ; \partial_{n^{*}} p^{\dagger}=\frac{-\gamma}{\left(n^{*}\right)^{2} Z^{\gamma+1}}<0 ; \partial_{n n^{*}} p^{\dagger}=\frac{-\gamma(\gamma+1)}{n^{2}\left(n^{*}\right)^{2} Z^{\gamma+2}}<0 ; \\
& \partial_{n n} p^{\dagger}=\frac{-2 \gamma}{n^{3} Z^{\gamma+1}}+\frac{\gamma(\gamma+1)}{n^{4} Z^{\gamma+2}}>0 ; \quad \partial_{n^{*} n^{*}} p^{\dagger}=\frac{2 \gamma}{\left(n^{*}\right)^{3} Z^{\gamma+1}}+\frac{\gamma(\gamma+1)}{\left(n^{*}\right)^{4} Z^{\gamma+2}}>0 ; \\
& \text { with } Z=\left(\frac{1}{n}-\frac{1}{n^{*}}\right),
\end{aligned}
$$

Then the Hessian matrix of $\tilde{p}^{\dagger}\left(n, n^{*}\right)$ is given by

$$
\tilde{H}\left(n, n^{*}\right)=\left(\begin{array}{cc}
\frac{-2 \gamma}{n^{3} Z^{\gamma+1}}+\frac{\gamma(\gamma+1)}{n^{4} Z^{\gamma+2}} & -\frac{\gamma(\gamma+1)}{n^{2} n^{* 2} Z^{\gamma+2}} \\
-\frac{\gamma(\gamma+1)}{n^{2} n^{* 2} Z^{\gamma+2}} & \frac{2 \gamma}{n^{* 3} Z^{\gamma+1}}+\frac{\gamma(\gamma+1)}{n^{* 4} Z^{\gamma+2}}
\end{array}\right)
$$

and its determinant is

$$
\operatorname{det}\left(\tilde{H}\left(n, n^{*}\right)\right)=\frac{2 \gamma}{n^{3} n^{* 3} Z^{\gamma+1}}\left(\frac{\gamma(\gamma+1)}{Z^{\gamma+1}}-\frac{2 \gamma}{Z^{\gamma+1}}\right) .
$$

For all $\gamma>1, \operatorname{det}\left(\tilde{H}\left(n, n^{*}\right)\right)>0$, therefore $\tilde{H}(n, u)$ is positive definite. Then, $H(n, u)$ is positive definite if

$$
\left(\frac{\partial p}{\partial n}\right)^{2} \frac{\partial p}{\partial n^{*}} \frac{d^{2} n^{*}}{d u^{2}} \geq 0 .
$$


The inequality (5.3) is satisfied since $\left(\frac{\partial p}{\partial n}\right)^{2} \geq 0, \frac{\partial p}{\partial n^{*}} \leq 0$ and due to the assumption (A-3), $\frac{d^{2} n^{*}}{d u^{2}} \leq 0$. Therefore, the eigenvalue $\lambda_{1}$ is genuinely non linear.

\section{Acknowledgments}

This work has been partially supported by the French ACI-NIM (Nouvelles Interactions des Mathématiques) $N^{o} 193$ (2004). S. Moutari and F. Berthelin would like to thank the Laboratoire MIP at University of Toulouse III for the support and the hospitality.

\section{References}

[1] A. Aw and M. Rascle, Resurection of second order models of traffic flow, SIAM J. Appl. Math., 60 (2000), 916-944.

[2] M. Bando and K. Hesebe and A. Nakayama and A. Shibata and Y. Sugiyama, Dynamical model of traffic congestion and numerical simulation, Phys. Rev. E, 51 (2) (1995), 1035-1042.

[3] F. Berthelin, Existence and weak stability for a two-phase model with unilateral constraint, Math. Models \& Methods in the Applied Sciences, 12 (2002), 249-272.

[4] P. Degond and M. Delitala, Modelling and simulation of vehicular traffic jam formation, Preprint., (2007).

[5] F. Berthelin, P. Degond, M. Delitala and M. Rascle, A model for the formation and evolution of traffic jams, Arch. Rat. Mechanics and Anal., (2005) (To appear).

[6] J. M. Greenberg, Extensions and amplifications of a traffic model of Aw and Rascle, SIAM J. Appl. Math., 62 (2001) 729-745.

[7] Y. Brenier, E. Grenier, Sticky particles and scalar conservation laws, SIAM J. Numer. Anal., 35 (1998) 2317-2328.

[8] R. M. Colombo, On a $2 \times 2$ hyperbolic traffic flow model, Math. Comp. modeling, 35 (2002) 683-688.

[9] C. M. Dafermos, Hyperbolic Conservation Laws in Continuum Physics, Springer Verlag, Berlin, Heidelberg, New York, 2000.

[10] C. F. Daganzo, Requiem for second order fluid approximations of traffic flow, Trans. Res. B, 29 (1995), 277-289.

[11] D. C. Gazis, R. Herman and R. Rothery Nonlinear follow-the-leader models of traffic flow, Operations Res., 9 (1961), 545-567. 
[12] D. Helbing, Improved fluid-dynamic model for vehicular traffic, Phys. Rev. E, 51 (1995), 3164 .

[13] R. Illner and A. Klar and M. Materne, Vlasov-Fokker-Planck models for multilane traffic flow, Commun. Math. Sci., 1 (2003), 1-12.

[14] A. Klar and R. Wegener Enskog-like Kinetic models for vehicular traffic, J. Stat. Phys., (1997), 91-114.

[15] R. D. Kühne, Macroscopic freeway model for dense traffic - stop-start waves and incident detection, In Proceedings of the 9th International Symposium on Transportation and Traffic Theory, Ed. I. Volmuller and R. Hamerslag, VNU Science Press, Utrecht, The Netherlands, 3 (1984), 21-42.

[16] P. D. Lax, Hyperbolic systems of conservation laws and the mathematical theory of shock waves, CBMF-NSF Regional Series in Applied Math. SIAM Philadelphia, (1973).

[17] M. J. Lighthill and J. B. Whitham, On kinematic waves: I. Flow movement in long rivers. II. A theory of traffic flow on long crowded roads, Proc. Roy. Soc., A229 (1955), 1749-1766.

[18] K. Nagel and M. Schreckenberg, A cellular automaton model for freeway traffic, J. Physique, 2 (1992), 2221-2229.

[19] P. Nelson, A kinetic model of vehicular traffic and its associated bimodal equilibruim solutions, Transp. Theory Stat. Phys., 24 (1995), 383-408.

[20] S. L. Paveri-Fontana, On Boltzmann-like treatments for traffic flow, Transportation Res., 9 (1975), 225-235.

[21] H. J. Payne, FREFLO: A macroscopic simulation model of freeway traffic, Transportation Research Record, 722 (1979), 68-75.

[22] R. Herman and I. Prigogine, Kinetic theory of vehicular traffic, American Elsevier publishing co, New-York 1971.

[23] P. I. Richards, Shock waves on the highway, Operations Research, 4 (1956), $42-51$.

[24] B. Temple, Systems of conservation laws with coinciding shock and rarefaction curves, Cotemp. Math., 17 (1983), 143-151.

[25] H. M. Zhang, A non-equilibrium traffic model devoid of gas-like behaviour, Trans. Res. B, 36 (2002), 275-298. 